\title{
Formality theorem for Hochschild cochains via transfer
}

\author{
Vasily Dolgushev
}

Belatedly to Simon Lyakhovich on the occasion of his 50th birthday.

\begin{abstract}
We construct a 2-colored operad $\mathbf{G e r}_{\infty}^{+}$which, on the one hand, extends the operad $\mathbf{G e r}_{\infty}$ governing homotopy Gerstenhaber algebras and, on the other hand, extends the 2-colored operad governing open-closed homotopy algebras (OCHA). We show that Tamarkin's $\mathbf{G e r}_{\infty}$-structure on the Hochschild cochain complex $C^{\bullet}(A, A)$ of an $A_{\infty^{-}}$ algebra $A$ extends naturally to a $\mathbf{G e r}_{\infty}^{+}$-structure on the pair $\left(C^{\bullet}(A, A), A\right)$. We show that a formality quasi-isomorphism for the Hochschild cochains of the polynomial algebra can be obtained via transfer of this $\mathbf{G e r}_{\infty}^{+}$-structure to the cohomology of the pair $\left(C^{\bullet}(A, A), A\right)$. We show that $\mathbf{G e r}_{\infty}^{+}$is a sub DG operad of the first sheet $E^{1}(\mathrm{SC})$ of the homology spectral sequence for the Fulton-MacPherson version SC of Voronov's Swiss Cheese operad. Finally, we prove that the DG operads $\mathbf{G e r}_{\infty}^{+}$and $E^{1}(\mathrm{SC})$ are non-formal.
\end{abstract}

Keywords: Operads, formality theorems, homotopy algebras.

2010 MSC: 18D50, 55U35.

\section{Contents}

1 Introduction

2 Preliminaries 3

2.1 Notation and conventions . . . . . . . . . . . . . . 3

2.2 Hochschild cochain complex of an $A_{\infty}$-algebra $\ldots \ldots \ldots \ldots$

3 The operad $\mathrm{Ger}_{\infty}^{+} \quad 7$

$4 \quad$ Algebras over the operad $\mathrm{Ger}_{\infty}^{+}$

$4.1 \mathrm{Ger}_{\infty}^{+}$versus the operad $\mathcal{O C}_{\infty}$ governing open-closed homotopy algebras (OCHA) 13

4.2 Homotopies of $\mathrm{Ger}_{\infty}^{+}$-algebra structures . . . . . . . . . . . . . . . 14

4.3 Transfer theorem . . . . . . . . . . . . . . . . . . . . 17

$4.4 \mathbf{G e r}_{\infty}^{+}$-algebra structure on $\left(C^{\bullet}(A, A), A\right) \ldots \ldots \ldots \ldots \ldots$

5 The formality quasi-isomorphism for $C^{\bullet}(A, A)$ via transfer of the $\mathrm{Ger}_{\infty^{-}}^{+}$ structure 
6 A Link between Ger $_{\infty}^{+}$and Voronov's Swiss Cheese operad SC 26

6.1 The Swiss Cheese operad SC . . . . . . . . . . . . . . . . 26

6.2 The operad $H_{\bullet}(\mathrm{SC})$ and the cooperads $H^{\bullet}(\mathrm{SC})$ and sc $\ldots \ldots \ldots . \ldots .28$

6.3 The first sheet $E^{1}(\mathrm{SC})$ of the spectral sequence for $\mathrm{SC} \ldots \ldots . \ldots$

7 The DG operads $\mathrm{Ger}_{\infty}^{+}$and $E^{1}(\mathrm{SC})$ are non-formal 32

8 Discussion $\quad 33$

\section{Introduction}

This paper is devoted to a natural 2-colored extension $\mathbf{G e r}_{\infty}^{+}$of the operad $\mathbf{G e r}_{\infty}$ governing homotopy Gerstenhaber algebras. Informally a $\mathbf{G e r}_{\infty}^{+}$-algebra is a blend of two ubiquitous algebraic structures: $A_{\infty}$-algebra and homotopy Gerstenhaber algebra. The notion of $A_{\infty^{-}}$ algebra was invented by J. Stasheff [38]. The notion of homotopy Gerstenhaber algebra has its origin in works [14], [16], [26] of M. Gerstenhaber, E. Getzler, and T. Kadeishvili. This latter notion was developed and formalized in works [1], [15], [19], [32], 35], 40], [45]. In this paper we describe interesting relations of the operad $\mathbf{G e r}_{\infty}^{+}$to Kontsevich's formality theorem [30], to the operad $\mathcal{O C}_{\infty}$ governing open-closed homotopy algebras [27], and to Voronov's Swiss Cheese operad [46].

We define the DG operad $\mathbf{G e r}_{\infty}^{+}$as the cobar construction of a cooperad $\mathbf{S}$ which is closely related to the cohomology cooperad $H^{\bullet}(\mathrm{SC})$ of Voronov's Swiss Cheese operad SC. We show that algebras over $\mathbf{G e r}_{\infty}^{+}$are pairs $(V, A)$ with the following data:

- $\mathrm{A} \mathrm{Ger}_{\infty}$-structure on $V$,

- An $A_{\infty}$-structure on $A$, and

- an $L_{\infty}$-morphism from $V$ to the Hochschild cochain complex $C^{\bullet}(A, A)$ of $A$.

Using this description we show that for every $A_{\infty}$-algebra $A$ Tamarkin's $\mathbf{G e r}_{\infty}$-structure on the Hochschild cochain complex $C^{\bullet}(A, A)$ extends naturally to a $\mathbf{G e r}_{\infty}^{+}$-structure on the pair $\left(C^{\bullet}(A, A), A\right)$.

We show that the DG operad $\mathrm{Ger}_{\infty}^{+}$satisfies the minimal model condition [39]. This condition implies that the operad $\mathbf{G e r}_{\infty}^{+}$is cofibrant and $\mathbf{G e r}_{\infty}^{+}$-structures can be transferred across quasi-isomorphisms.

Using Tamarkin's rigidity (see Theorem 5.5) for the Hochschild cohomology $H H^{\bullet}(A, A)$ of the polynomial algebra $A=\mathbb{K}\left[x^{1}, \ldots, x^{d}\right]$ we show that a formality quasi-isomorphism for Hochschild cochains $C^{\bullet}(A, A)$ coincides with the corresponding part of the transferred Ger $_{\infty}^{+}$-structure on the pair $\left(H H^{\bullet}(A, A), A\right)$.

Furthermore, we show that the operad $\mathrm{Ger}_{\infty}^{+}$allows us to establish a necessary and sufficient condition (Theorem 5.4) for an abstract $L_{\infty}$ morphism

$$
H H^{\bullet}(A, A) \succ \rightarrow C^{\bullet}(A, A)
$$

to be homotopic to an $L_{\infty}$ quasi-isomorphism obtained via Tamarkin's procedure [10], 21], [40]. 
We also show that $\mathrm{Ger}_{\infty}^{+}$is a sub-operad of the first sheet $E^{1}(\mathrm{SC})$ of the homology spectral sequence for the topological Swiss Cheese operad considered by A. Voronov in [46]. Finally we prove that the DG operads $\mathrm{Ger}_{\infty}^{+}$and $E^{1}(\mathrm{SC})$ are non-formal.

The paper is organized as follows.

In Section 2 we fix notation and conventions. In this section we also recall necessary facts about Hochschild cochain complex of an $A_{\infty}$-algebra. In Section 3 we introduce the operad $\mathbf{G e r}_{\infty}^{+}$and define $\mathbf{G e r}_{\infty}^{+}$-morphism as well as $\mathbf{G e r}_{\infty}^{+}$quasi-isomorphisms. In Section 4 we give an alternative description of $\mathbf{G e r}_{\infty}^{+}$-algebras and show that the operad $\mathbf{G e r}_{\infty}^{+}$ is an extension of the operad $\mathcal{O C}_{\infty}$ governing open-closed homotopy algebras (OCHA). In this section we also discuss homotopies of $\mathrm{Ger}_{\infty}^{+}$-algebras, prove the transfer theorem, and construct a $\mathbf{G e r}_{\infty}^{+}$-algebra structure on the pair $\left(C^{\bullet}(A, A), A\right)$. In Section 5 we establish a necessary and sufficient condition for an abstract $L_{\infty}$-morphism from $H H^{\bullet}(A, A)$ to $C^{\bullet}(A, A)$ to be homotopic to the one obtained via Tamarkin's procedure [10, [21], [40]. In this section we also show that a formality quasi-isomorphism for Hochschild cochains $C^{\bullet}(A, A)$ of the polynomial algebra $A=\mathbb{K}\left[x^{1}, \ldots, x^{d}\right]$ is obtained via transfer of the $\mathbf{G e r}_{\infty}^{+}$-algebra on $\left(C^{\bullet}(A, A), A\right)$ to the cohomology. In Section 6 we describe the first sheet of the homology spectral sequence $E^{1}(\mathrm{SC})$ for the Fulton-MacPherson version SC of Voronov's Swiss Cheese operad [46]. In this section we show that $\mathbf{G e r}_{\infty}^{+}$is a suboperad of the DG operad $E^{1}(\mathrm{SC})$. In Section 7 we prove that the DG operads $\mathrm{Ger}_{\infty}^{+}$and $E^{1}(\mathrm{SC})$ are non-formal. Finally, in the concluding section, we discuss a few open questions.

\section{Acknowledgment}

I was secretly introduced to the fascinating world of homotopy algebras when I studied quantization of gauge systems with my advisor Simon Lyakhovich in Tomsk State University. I would like to thank Simon for his guidance and greet him with his anniversary. I would like to thank S. Shadrin, J. Stasheff, B. Vallette, A. Voronov and T. Willwacher for useful discussions. I am also thankful to J. Stasheff for his remarks on the first draft of the paper. I would like to thank the anonymous referee for carefully reading my manuscript and for many useful suggestions. A large part of this paper was written when I was an assistant professor at the UC Riverside where I had excellent working conditions and great colleagues. I would like to thank anonymous taxpayers of California for supporting the UC system and my work at UC Riverside. The results of this paper were presented at the Southern California Algebra Conference in UCLA and at the Geometry and Physics seminar at Northwestern University. I would like to thank participants of the conference and the seminar for questions and useful comments. I am partially supported by the NSF grant DMS 0856196, Regent's Faculty Fellowship, and the Grant for Support of Scientific Schools NSh-3036.2008.2.

\section{Preliminaries}

\subsection{Notation and conventions}

The ground field $\mathbb{K}$ has the zero characteristic. Depending on a context our underlying symmetric monoidal category is either the category of graded vector spaces, or the category of cochain complexes, or the category of compactly generated topological spaces. By suspension 
$\mathbf{s} V$ of a graded vector space (or a chain complex) $V$ we mean $\varepsilon \otimes V$, where $\varepsilon$ is a onedimensional vector space placed in degree +1 . For a vector $v \in V$ we denote by $|v|$ its degree. $S_{n}$ denotes the symmetric group on $n$ letters and $\mathrm{Sh}_{p, q}$ denotes the subset of $(p, q)$ shuffles in $S_{p+q}$.

For a graded vector space (or a cochain complex) $V$ the notation $S(V)$ (resp. $\underline{S}(V)$ ) is reserved for the symmetric algebra (resp. truncated symmetric algebra) of $V$ :

$$
\begin{gathered}
S(V)=\mathbb{K} \oplus V \oplus S^{2}(V) \oplus S^{3}(V) \oplus \ldots, \\
\underline{S}(V)=V \oplus S^{2}(V) \oplus S^{3}(V) \oplus \ldots .
\end{gathered}
$$

Similarly, $T(V)$ (resp. $\underline{T}(V))$ denotes the tensor algebra (resp. truncated tensor algebra) of $V$ :

$$
\begin{gathered}
T(V)=\mathbb{K} \oplus V \oplus V^{\otimes 2} \oplus V^{\otimes 3} \oplus \ldots, \\
\underline{T}(V)=V \oplus V^{\otimes 2} \oplus V^{\otimes 3} \oplus \ldots .
\end{gathered}
$$

For an operad $\mathcal{O}$ (resp. a cooperad $\mathcal{C}$ ) and a cochain complex $V$ we denote by $\mathcal{O}(V)$ (resp. $\mathcal{C}(V))$ the free $\mathcal{O}$-algebra generated by $V$ (resp. the cofree $\mathcal{C}$-coalgebra cogenerated by $V)$. The notation $\operatorname{Cobar}(\mathcal{C})$ is reserved for the cobar construction [13, [19] of a cooperad $\mathcal{C}$.

By "suspension" of a (co)operad $\mathcal{O}$ we mean the (co)operad $\Lambda(\mathcal{O})$ whose $m$-th space is

$$
\Lambda(\mathcal{O})(m)=\mathbf{s}^{1-m} \mathcal{O}(m) \otimes \operatorname{sgn}_{m},
$$

where $\operatorname{sgn}_{m}$ is the sign representation of the symmetric group $S_{m}$. For example, a $\Lambda$ Liealgebra is nothing but a Lie algebra with the bracket of degree -1 . Similarly $\Lambda$ coassoc is the cooperad governing coassociative coalgebras with comultiplication of degree -1 .

It is not hard to see that for every (co)operad $\mathcal{O}$ and for every graded vector space $V$ we have the isomorphism

$$
\Lambda(\mathcal{O})(V)=\mathbf{s} \mathcal{O}\left(\mathbf{s}^{-1} V\right) .
$$

It is important to make a remark about degrees of cooperations in coalgebras over cooperads. If $\mathcal{C}$ is a cooperad and $v$ is a homogeneous vector in $\mathcal{C}(m)$ then the corresponding cooperation

$$
A \rightarrow A^{\otimes m}
$$

on a $\mathcal{C}$-coalgebra $A$ has the degree $-|v|$. In this paper by degree of a cooperation we mean the degree of the corresponding vector in the cooperad but not the degree of the map. For example, a vector $\Delta \in \Lambda \operatorname{coassoc}(2)$ has the degree -1 but the corresponding map

$$
\Delta: A \rightarrow A \otimes A
$$

for a $\Lambda$ coassoc-coalgebra $A$ has the degree 1 .

In this paper we often encounter $L_{\infty}$-algebras (resp. (DG) Lie algebras) with the binary

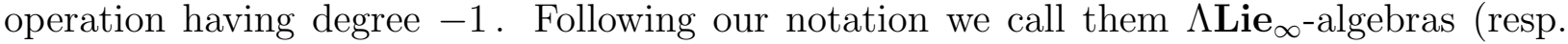
$\Lambda$ Lie-algebras). However, we still retain the word " $L_{\infty}$-morphism" for a morphism in the category of $\Lambda \mathbf{L i e}_{\infty}$-algebras.

Thus a $\Lambda \mathbf{L i e} \mathbf{\infty}_{\infty}$-structure on a graded vector space $\mathcal{L}$ is a degree 1 codifferential $\hat{Q}_{\mathcal{L}}$ on the cofree $\Lambda^{2}$ cocomm coalgebra

$$
\Lambda^{2} \operatorname{cocomm}(\mathcal{L})=\mathbf{s}^{2} S\left(\mathbf{s}^{-2} \mathcal{L}\right) .
$$


Similarly, an $L_{\infty}$-morphism $\hat{U}$ from a $\Lambda \mathbf{L i e}_{\infty}$-algebra $\mathcal{L}$ to a $\Lambda \mathbf{L i e}_{\infty}$-algebra $\widetilde{\mathcal{L}}$ is a morphism of $\Lambda^{2}$ cocomm-coalgebras

$$
\hat{U}: \mathbf{s}^{2} S\left(\mathbf{s}^{-2} \mathcal{L}\right) \rightarrow \mathbf{s}^{2} S\left(\mathbf{s}^{-2} \widetilde{\mathcal{L}}\right)
$$

compatible with the codifferentials.

Since $\hat{U}$ is a morphism to a cofree coalgebra, it is uniquely determined by its composition

$$
U=p \circ \hat{U}: \mathbf{s}^{2} S\left(\mathbf{s}^{-2} \mathcal{L}\right) \rightarrow \widetilde{\mathcal{L}}
$$

with the natural projection

$$
p: \mathbf{s}^{2} S\left(\mathbf{s}^{-2} \widetilde{\mathcal{L}}\right) \rightarrow \widetilde{\mathcal{L}} .
$$

The notation Ger is reserved for the operad governing Gerstenhaber algebras [14, [19] and $\mathbf{G e r}^{\vee}$ denotes the corresponding Koszul dual [13], [19], [20] cooperad. This cooperad can be obtained from the linear dual Ger* of Ger by applying the operation $\Lambda$ twice:

$$
\operatorname{Ger}^{\vee}=\Lambda^{2} \text { Ger }^{*}
$$

Following [13], [19], and [20] the DG operad Cobar $\left(\mathbf{G e r}^{\vee}\right)$ can be chosen as a model for the operad which governs homotopy Gerstenhaber algebras. So, in this paper, we set

$$
\operatorname{Ger}_{\infty}=\operatorname{Cobar}\left(\mathbf{G e r}^{\vee}\right)
$$

For colored (co)operads we use notation and conventions from paper [3] by C. Berger and I. Moerdijk. All colored (co)operads in this paper are 2-colored and we denote the two colors by $\mathfrak{c}$ and $\mathfrak{o}$. Furthermore, we fix the order $\mathfrak{c}<\mathfrak{o}$ on the set $\{\mathfrak{c}, \mathfrak{o}\}$. Hence, due to [3], Remark 1.3], every 2-colored (co)operad $\mathcal{O}$ is completely determined by objects

$$
\begin{gathered}
\mathcal{O}(\underbrace{\mathfrak{c}, \mathfrak{c}, \ldots, \mathfrak{c}}_{k}, \underbrace{\mathfrak{o}, \mathfrak{o}, \ldots, \mathfrak{o}}_{n} ; \mathfrak{c}), \\
\mathcal{O}(\underbrace{\mathfrak{c}, \mathfrak{c}, \ldots, \mathfrak{c}}_{k}, \underbrace{\mathfrak{o}, \mathfrak{o}, \ldots, \mathfrak{o}}_{n} ; \mathfrak{o})
\end{gathered}
$$

together with the right action of the group $S_{k} \times S_{n}$ and by the corresponding (co)operadic (co)multiplications for these objects.

We often use the following short-hand notation for objects of 2-colored (co)operads

$$
\begin{aligned}
\mathcal{O}^{\mathfrak{c}}(n, k) & :=\mathcal{O}(\underbrace{\mathfrak{c}, \mathfrak{c}, \ldots, \mathfrak{c}}_{n}, \underbrace{\mathfrak{o}, \mathfrak{o}, \ldots, \mathfrak{o}}_{k} ; \mathfrak{c}), \\
\mathcal{O}^{\mathfrak{o}}(n, k) & :=\mathcal{O}(\underbrace{\mathfrak{c}, \mathfrak{c}, \ldots, \mathfrak{c}}_{n}, \underbrace{\mathfrak{o}, \mathfrak{o}, \ldots, \mathfrak{o}}_{k} ; \mathfrak{o}) .
\end{aligned}
$$

Let us consider, for example, the operad Lie whose algebras are Lie algebras (in the category of vector spaces). This operad can be upgraded to the 2-colored operad $\mathbf{L i e}^{+}$whose algebras are pairs $(\mathcal{V}, \mathcal{W})$ where $\mathcal{V}$ is a Lie algebra and $\mathcal{W}$ is a module over $\mathcal{W}$. To vectors in $\mathcal{V}$ (resp, $\mathcal{W}$ ) we assign the color $\mathfrak{c}\left(\right.$ resp. o). It is not hard to see that for $\mathbf{L i e}^{+}$we have

$$
\begin{aligned}
\left(\mathbf{L i e}^{+}\right)^{\mathfrak{c}}(n, 0) & =\mathbf{L i e}(n), \\
\left(\mathbf{L i e}^{+}\right)^{\mathfrak{o}}(n, 1) & =\mathbb{K}\left[S_{n}\right],
\end{aligned}
$$

and the remaining vector spaces are $\mathbf{0}$. 
Remark 2.1 For colored operads of cochain complexes we use a bit of terminology from closed model categories. Thus, following [2] and [3], we call a map $f: \mathcal{O} \rightarrow \widetilde{\mathcal{O}}$ of colored operads a fibration if for every collection of colors $c_{1}, \ldots, c_{k}, c$ the map

$$
f: \mathcal{O}\left(c_{1}, \ldots, c_{k} ; c\right) \rightarrow \widetilde{\mathcal{O}}\left(c_{1}, \ldots, c_{k} ; c\right)
$$

is surjective. We say that $f: \mathcal{O} \rightarrow \widetilde{\mathcal{O}}$ is an acyclic fibration is $f$ is a quasi-isomorphism and a fibration. A map $f: \mathcal{O} \rightarrow \widetilde{\mathcal{O}}$ is called a cofibration if $f$ satisfies the left lifting property with respect to all acyclic fibrations. Finally, a colored operad $\mathcal{O}$ is called cofibrant if the unique map $* \rightarrow \mathcal{O}$ from the initial object $*$ is a cofibration.

Unfortunately, to the best of our knowledge, it is not yet established whether the category of all colored operads with the above (co)fibrations is a closed model category. For this reason, in our paper, we use the above terminology but not the full power of closed model categories.

We use the reversed grading on each homological complex. In particular, the homology groups $H_{\bullet}(X)$ of a topological space $X$ are concentrated in non-positive degrees and the Poincaré duality takes this unusual form:

$$
H_{\bullet}(X) \cong H^{\operatorname{dim} X+\bullet}(X) .
$$

Similarly, for the homology spectral sequence associated to an increasing filtration $F^{p-1} X \subset$ $F^{p} X \subset \ldots$ on a space $X$ we have

$$
E_{p, q}^{1}(X)=H_{p+q}\left(F^{-p} X, F^{-p-1} X\right)
$$

and

$$
d_{1}: E_{p, q}^{1}(X) \rightarrow E_{p+1, q}^{1}(X)
$$

\subsection{Hochschild cochain complex of an $A_{\infty}$-algebra}

It is known that for every graded vector space $A$

$$
\bigoplus_{k \geq 0} \mathrm{~s}^{k} \operatorname{Hom}\left(A^{\otimes k}, A\right)
$$

is equipped with a $\Lambda$ Lie-algebra structure. The corresponding bracket is the Gerstenhaber bracket $[,]_{G}$. It is given by the formula

$$
\begin{gathered}
{\left[Q_{1}, Q_{2}\right]_{G}=} \\
\sum_{i=1}^{k_{1}}(-1)^{\varepsilon_{i, k_{2}}} Q_{1}\left(a_{1}, \ldots, Q_{2}\left(a_{i}, \ldots, a_{i+k_{2}-1}\right), \ldots, a_{k_{1}+k_{2}-1}\right)-(-1)^{\left(k_{1}+1\right)\left(k_{2}+1\right)}(1 \leftrightarrow 2),
\end{gathered}
$$

where $k_{1}$ (resp. $\left.k_{2}\right)$ is the degree of $Q_{1}\left(\operatorname{resp} . Q_{2}\right)$ in $(2.9)$ and

$$
\varepsilon_{i, k_{2}}=\left(k_{2}+1\right)\left(\left|a_{1}\right|+\cdots+\left|a_{i-1}\right|+i-1\right) .
$$

Let us recall that an $A_{\infty}$-algebra structure on $A$ is an element

$$
m \in \bigoplus_{k \geq 1} \mathbf{s}^{k} \operatorname{Hom}\left(A^{\otimes k}, A\right)
$$


satisfying the Maurer-Cartan equation:

$$
[m, m]_{G}=0
$$

Equation (2.12) implies that the formula

$$
\partial^{\text {Hoch }}=[m, \quad]_{G}
$$

defines a differential on the $\Lambda$ Lie-algebra (2.9) . In particular, restricting $m$ to $A$ we get a differential on $A$.

The cochain complex (2.9) with the differential (2.13) is called the Hochschild cochain complex of the $A_{\infty}$-algebra $(A, m)$. In this paper we denote this cochain complex by $C^{\bullet}(A, A)$.

\section{The operad $\mathrm{Ger}_{\infty}^{+}$}

Let us start the construction of the operad $\mathbf{G e r}_{\infty}^{+}$with the following definition

Definition 3.1 An $\mathbf{S}$-coalgebra is a pair of $\mathbf{G e r}^{\vee}$-coalgebra $V$ and a $\Lambda$ coassoc-coalgebra $A$ together with a unary operation

$$
\rho: A \rightarrow V
$$

of degree $\mathbb{1}^{1}-1$ such that the composition of the cobracket $\delta_{\mathfrak{c}}$ with $\rho$ is zero:

$$
\delta_{\mathfrak{c}} \circ \rho(a)=0,
$$

and the following diagrams commute:
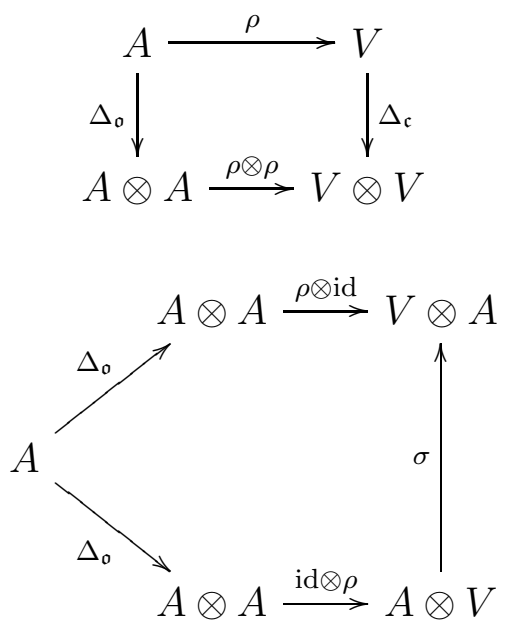

where $\Delta_{\mathfrak{c}}\left(\right.$ resp. $\left.\Delta_{\mathfrak{o}}\right)$ is the comultiplication in $V$ (resp. in A) and

$$
\sigma: A \otimes V \rightarrow V \otimes A
$$

is the mapping which switches tensor components.

\footnotetext{
${ }^{1}$ Recall that by degree of $\rho$ we mean the degree of the corresponding vector in the cooperad governing S-coalgebras.
} 
Remark 3.2 Diagram (3.2) says that $\rho$ is a map of coassociative coalgebras. However, the degrees of comultiplications $\Delta_{\mathfrak{c}}$ and $\Delta_{\mathfrak{o}}$ are different. This is why $\rho$ is forced to have degree -1 .

Composing the comultiplication $\Delta_{\mathfrak{o}}$ with $\rho \otimes$ id and id $\otimes \rho$ we get the maps

$$
\mu_{l}=\rho \otimes \operatorname{id} \circ \Delta_{\mathfrak{o}}: A \rightarrow V \otimes A
$$

and

$$
\mu_{r}=\mathrm{id} \otimes \rho \circ \Delta_{\mathfrak{o}}: A \rightarrow A \otimes V,
$$

respectively.

Using the axioms of $\mathbf{S}$-coalgebras it is not hard to show that

Proposition 3.3 If we view $V$ as a coassociative coalgebra then the maps $\mu_{l}$ (3.4) and $\mu_{r}$ (3.5) are left and right comodule structures on $A$ over $V$, respectively.

Remark 3.4 Axiom (3.3) implies that the comodule structure $\mu_{r}$ is obtained from $\mu_{l}$ via switching the tensor factors.

Proof. To prove that $\mu_{l}$ is a left comodule structure we need to show that the diagram

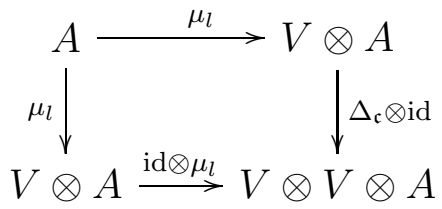

commutes.

The latter follows easily from the commutativity of the diagram below:

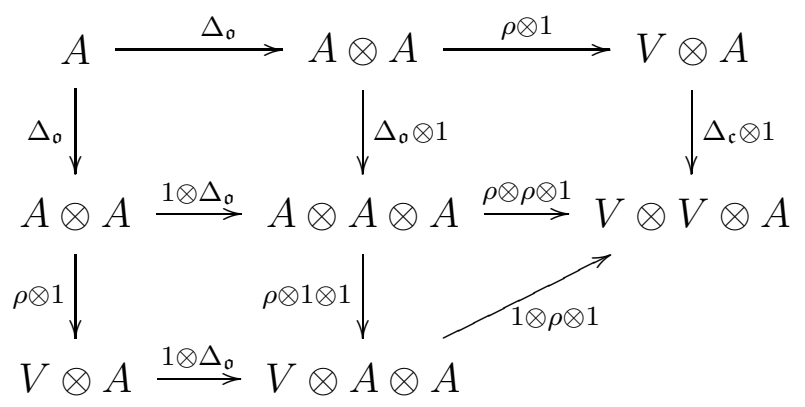

The corresponding statement for $\mu_{r}$ can be proved in a similar way.

Let us denote by $\mathbf{S}$ the cooperad which governs the $\mathbf{S}$-coalgebras. This is a 2-colored cooperad. We reserve the color $\mathfrak{c}$ for vectors in $V$ and the color $2 \mathfrak{o}$ for vectors in $A$. In Subsection 6.2 we describe a link between the cooperad $\mathbf{S}$ and the cohomology cooperad $H^{\bullet}(\mathrm{SC})$ of Voronov's Swiss Cheese operad SC .

The cofree $\mathbf{S}$-coalgebra $\mathbf{S}(V, A)$ cogenerated by the pair $(V, A)$ is the direct sum

$$
\mathbf{S}(V, A)=\mathbf{S}^{\mathfrak{c}}(V, A) \oplus \mathbf{S}^{\mathfrak{o}}(V, A)
$$

\footnotetext{
${ }^{2}$ This notation for colors comes from string theory: $\mathfrak{c}$ stands for closed strings and $\mathfrak{o}$ stands for open strings.
} 
where

$$
\mathbf{S}^{\mathfrak{c}}(V, A)=\operatorname{Ger}^{\vee}(V)
$$

and

$$
\mathbf{S}^{\mathfrak{o}}(V, A)=\mathbf{s}\left(\underline{S}\left(\mathbf{s}^{-2} V\right) \otimes T\left(\mathbf{s}^{-1} A\right)\right) \oplus \mathbf{s} \underline{T}\left(\mathbf{s}^{-1} A\right),
$$

where $\underline{T}\left(\mathbf{s}^{-1} A\right)$ (resp. $\left.\underline{S}\left(\mathbf{s}^{-2} V\right)\right)$ is the truncated tensor algebra of $\mathbf{s}^{-1} A$ (resp. the truncated symmetric algebra of $\left.\mathbf{s}^{-2} V\right)$.

The main hero of this article is the DG operad Cobar $(\mathbf{S})$. Since Cobar $(\mathbf{S})$ extends the operad governing homotopy Gerstenhaber algebras we denote it by $\mathbf{G e r}_{\infty}^{+}$.

Let us recall that, due to Proposition 2.15 in [19], a $\mathbf{G e r}_{\infty}^{+}$-algebra structure on $(V, A)$ is a degree 1 codifferential $\hat{Q}$ of the cofree coalgebra $\mathbf{S}(V, A)$.

This observation motivates the definition of a $\mathrm{Ger}_{\infty}^{+}$-morphism.

Definition 3.5 Let $(V, A)$ and $\left(V^{\prime}, A^{\prime}\right)$ be two $\mathbf{G e r}_{\infty}^{+}$-algebras. A $\mathbf{G e r}_{\infty}^{+}$-morphism from $(V, A)$ to $\left(V^{\prime}, A^{\prime}\right)$ is a morphism of $\mathbf{S}$-coalgebras

$$
\hat{T}: \mathbf{S}(V, A) \rightarrow \mathbf{S}\left(V^{\prime}, A^{\prime}\right)
$$

with the codifferentials $\hat{Q}$ and $\hat{Q}^{\prime}$ corresponding to the $\mathbf{G e r}_{\infty}^{+}$-structures on $(V, A)$ and $\left(V^{\prime}, A^{\prime}\right)$, respectively.

Since $\mathbf{S}\left(V^{\prime}, A^{\prime}\right)$ is cofree every morphism

$$
\hat{T}: \mathbf{S}(V, A) \rightarrow \mathbf{S}\left(V^{\prime}, A^{\prime}\right)
$$

of $\mathbf{S}$-coalgebras is uniquely determined by its composition

$$
T=p \circ \hat{T}: \mathbf{S}(V, A) \rightarrow V^{\prime} \oplus A^{\prime}
$$

with the projection $p: \mathbf{S}\left(V^{\prime}, A^{\prime}\right) \rightarrow V^{\prime} \oplus A^{\prime}$.

It is not hard to see that the compatibility of $\hat{T}$ with the codifferentials $\hat{Q}$ and $\hat{Q}^{\prime}$ implies that the restrictions:

$$
\left.T\right|_{V}: V \rightarrow V^{\prime}
$$

and

$$
\left.T\right|_{A}: A \rightarrow A^{\prime}
$$

are morphisms of cochain complexes.

This observation motivates the definition of $\mathrm{Ger}_{\infty}^{+}$quasi-isomorphism

Definition 3.6 $A$ Ger Ge $_{\infty}^{+}$quasi-morphism from $(V, A)$ to $\left(V^{\prime}, A^{\prime}\right)$ is a morphism of $\mathbf{S}$-coalgebras

$$
\hat{T}: \mathbf{S}(V, A) \rightarrow \mathbf{S}\left(V^{\prime}, A^{\prime}\right)
$$

for which the restrictions (3.11), (3.12) are quasi-isomorphisms of cochain complexes. 


\section{Algebras over the operad $\mathrm{Ger}_{\infty}^{+}$}

The following theorem gives us an alternative description ${ }^{3}$ of algebras over the operad $\mathbf{G e r}_{\infty}^{+}$.

Theorem 4.1 $A$ Ger $_{\infty}^{+}$-algebra structure on the pair of cochain complexes $(V, A)$ is a triple:

- $A \mathrm{Ger}_{\infty}$-structure on $V$,

- An $A_{\infty}$-structure on $A$, and

- An $L_{\infty}$-morphism from $V$ to the Hochschild cochain complex $C^{\bullet}(A, A)$ of the $A_{\infty}$ algebra $A$.

Proof. A $\mathrm{Ger}_{\infty}^{+}$-algebra structure on $(V, A)$ is a degree 1 codifferential $\hat{Q}$ on the cofree coalgebra $\mathbf{S}(V, A)$. This differential splits into the $\mathfrak{c}$-component $\hat{Q}^{\mathfrak{c}}$

$$
\hat{Q}^{\mathfrak{c}}: \operatorname{Ger}^{\vee}(V) \rightarrow \operatorname{Ger}^{\vee}(V)
$$

and the $\mathfrak{o}$-component

$$
\hat{Q}^{\mathfrak{o}}: \mathbf{S}^{\mathfrak{o}}(V, A) \rightarrow \mathbf{S}^{\mathfrak{o}}(V, A) .
$$

Since $\mathbf{S}(V, A)$ is cofree, the codifferential $\hat{Q}$ is uniquely determined by its composition

$$
Q=p \circ \hat{Q}: \mathbf{S}(V, A) \rightarrow V \oplus A
$$

with the projection

$$
p: \mathbf{S}(V, A) \rightarrow V \oplus A .
$$

The composition $Q$ also splits into the $\mathfrak{c}$-component

$$
Q^{\mathfrak{c}}: \operatorname{Ger}^{\vee}(V) \rightarrow V
$$

and the $\mathfrak{o}$-component

$$
Q^{\mathfrak{o}}: \mathbf{s}\left(\underline{S}\left(\mathbf{s}^{-2} V\right) \otimes T\left(\mathbf{s}^{-1} A\right)\right) \oplus \mathbf{s} \underline{T}\left(\mathbf{s}^{-1} A\right) \rightarrow A .
$$

We claim that $Q^{\mathfrak{c}}$ (4.4) gives us a $\mathbf{G e r}_{\infty}$-algebra structure on $V$, the restriction

$$
\left.Q^{\mathfrak{o}}\right|_{\mathbf{s} \underline{T}\left(\mathbf{s}^{-1} A\right)}: \mathbf{s} \underline{T}\left(\mathbf{s}^{-1} A\right) \rightarrow A
$$

gives us an $A_{\infty}$-structure on $A$ and the restriction

$$
\left.Q^{\mathfrak{o}}\right|_{\mathbf{s}\left(\underline{S}\left(\mathbf{s}^{-2} V\right) \otimes T\left(\mathbf{s}^{-1} A\right)\right)}: \mathbf{s}\left(\underline{S}\left(\mathbf{s}^{-2} V\right) \otimes T\left(\mathbf{s}^{-1} A\right)\right) \rightarrow A
$$

gives us an $L_{\infty}$-morphism from the $\Lambda \mathbf{L i e}_{\infty}$-algebra $V$ to the $\Lambda$ Lie-algebra $C^{\bullet}(A, A)$.

The first two statements are obvious. Indeed, the map $Q^{\mathfrak{c}}$ (4.4) corresponds to the codifferential $\hat{Q}^{\mathfrak{c}}$ on the $\operatorname{Ger}^{\vee}$-coalgebra $\operatorname{Ger}^{\vee}(V)$. This is a $\mathbf{G e r}_{\infty}$-structure on $V$. The map (4.6) corresponds to the codifferential

$$
\left.\hat{Q}^{\mathrm{o}}\right|_{\mathbf{s} \underline{T}\left(\mathbf{s}^{-1} A\right)}: \mathbf{s} \underline{T}\left(\mathbf{s}^{-1} A\right) \rightarrow \mathbf{s} \underline{T}\left(\mathbf{s}^{-1} A\right)
$$

\footnotetext{
${ }^{3}$ I would like to thank Thomas Willwacher who suggested to me this alternative description.
} 
on the cofree $\Lambda$ coassoc-coalgebra

$$
\Lambda \operatorname{coassoc}(A)=\mathbf{s} \underline{T}\left(\mathbf{s}^{-1} A\right) .
$$

Thus we get an $A_{\infty}$-algebra structure on $A$.

To prove the third statement we replace the $\mathfrak{o}$-component $\mathbf{S}^{\mathfrak{o}}(V, A)$ of the coalgebra $\mathbf{S}(V, A)$ by its desuspension

$$
\mathbf{s}^{-1} \mathbf{S}^{\mathfrak{o}}(V, A)=\underline{S}\left(\mathbf{s}^{-2} V\right) \otimes T\left(\mathbf{s}^{-1} A\right) \oplus \underline{T}\left(\mathbf{s}^{-1} A\right)
$$

and conjugate the $\mathfrak{o}$-component $\hat{Q}^{\mathfrak{o}}$ of the codifferential $\hat{Q}$ by the suspension isomorphism. (Thanks to this technical modification we will have simpler expressions for sings in our formulas.) We will use the same notation $Q^{\mathfrak{o}}$ for this conjugated map:

$$
Q^{\mathfrak{o}}:\left(\underline{S}\left(\mathbf{s}^{-2} V\right) \otimes T\left(\mathbf{s}^{-1} A\right)\right) \oplus \underline{T}\left(\mathbf{s}^{-1} A\right) \rightarrow \mathbf{s}^{-1} A .
$$

Due to the above shift the comultiplication $\Delta_{\mathfrak{o}}$ on (4.8) has degree 0 and the explicit formula for $\Delta_{\mathfrak{o}}$ reads

$$
\begin{gathered}
\Delta_{\mathfrak{o}}\left(v_{1}, \ldots, v_{k} ; a_{1}, \ldots, a_{n}\right)= \\
\sum_{(p, t) \in E_{k, n}} \sum_{\substack{\lambda \in \operatorname{Sh}_{p, k-p} \\
\left(v_{\lambda(p+1)}, \ldots, v_{\lambda(k)} ; a_{t+1}, \ldots, a_{n}\right)}}(-1)^{\eta_{p, t}^{\lambda}}\left(v_{\lambda(1)}, \ldots, v_{\lambda(p)} ; a_{1}, \ldots, a_{t}\right) \otimes
\end{gathered}
$$

where

$$
\begin{gathered}
E_{k, n}=\{0,1, \ldots, k\} \times\{0,1, \ldots, n\} \backslash\{(0,0),(k, n)\} \\
\eta_{p, t}^{\lambda}=\sum_{i<j}\left|v_{i}\right|\left|v_{j}\right|+\sum_{i=1}^{t} \sum_{j=p+1}^{k}\left|v_{\lambda(j)}\right|\left(\left|a_{i}\right|+1\right) .
\end{gathered}
$$

Using the compatibility of $\hat{Q}$ with the S-coalgebra structure one deduces readily a formula for $\hat{Q}^{\mathfrak{o}}$ in terms of $Q^{\mathfrak{c}}$ and $Q^{\mathfrak{o}}$ :

$$
\begin{gathered}
\hat{Q}^{\mathfrak{o}}\left(v_{1}, \ldots, v_{k} ; a_{1}, \ldots, a_{n}\right)= \\
\sum_{p=1}^{k} \sum_{\lambda \in \operatorname{Sh}_{p, k-p}}(-1)^{\varepsilon_{\lambda}}\left(Q^{\mathfrak{c}}\left(v_{\lambda(1)}, \ldots, v_{\lambda(p)}\right), v_{\lambda(p+1)}, \ldots, v_{\lambda(k)} ; a_{1}, \ldots, a_{n}\right)+ \\
\sum_{1 \leq t \leq s \leq n}^{0 \leq p \leq k} \sum_{\substack{1 \leq t \leq n+1 \\
\text { where }}}(-1)^{\varepsilon_{\lambda}+\varepsilon_{t, p}^{\lambda}}\left(v_{\lambda(1)}, \ldots, v_{\lambda(p)} ; a_{1}, \ldots, Q^{\mathfrak{o}}\left(v_{\lambda(p+1)}, \ldots, v_{\lambda(k)} ; a_{t}, \ldots, a_{s}\right) \ldots, a_{n}\right)+ \\
(-1)^{\varepsilon_{\lambda}+\varepsilon_{t, p}^{\lambda}}\left(v_{\lambda(1)}, \ldots, v_{\lambda(p)}, ; a_{1}, \ldots, a_{t-1}, Q^{\mathfrak{o}}\left(v_{\lambda(p+1)}, \ldots, v_{\lambda(k)}\right), a_{t}, \ldots, a_{n}\right) \\
\varepsilon_{\lambda}=\sum_{i<j}\left|\sum_{\lambda(i)>\lambda(j)}\right| v_{i}|| v_{j} \mid
\end{gathered}
$$


and

$$
\varepsilon_{t, p}^{\lambda}=\sum_{j=1}^{p}\left|v_{\lambda(j)}\right|+\sum_{j=p+1}^{k} \sum_{l=1}^{t-1}\left|v_{\lambda(j)}\right|\left(\left|a_{l}\right|+1\right)+\sum_{l=1}^{t-1}\left(\left|a_{l}\right|+1\right) .
$$

Restricting the codifferential $\hat{Q}^{\mathfrak{c}}$ to the coalgebra

$$
\Lambda^{2} \operatorname{cocomm}(V) \subset \operatorname{Ger}^{\vee}(V)
$$

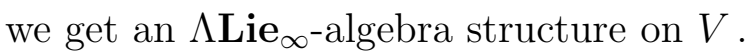

An $L_{\infty}$-morphism from $V$ to $C^{\bullet}(A, A)$ is a degree 0 map

$$
U: \Lambda^{2} \operatorname{cocomm}(V) \rightarrow C^{\bullet}(A, A)
$$

satisfying the coherence equations

$$
\begin{gathered}
\sum_{p=1}^{k} \sum_{\lambda \in \mathrm{Sh}_{p, k-p}}(-1)^{\varepsilon_{\lambda}} U\left(Q^{\mathfrak{c}}\left(v_{\lambda(1)}, \ldots, v_{\lambda(p)}\right), v_{\lambda(p+1)}, \ldots, v_{\lambda(k)}\right) \\
=-\left[m, U\left(v_{1}, \ldots, v_{k}\right)\right]_{G}- \\
\sum_{p=1}^{k-1} \sum_{\lambda \in \operatorname{Sh}_{p, k-p}}(-1)^{\varepsilon_{\lambda}+\left|v_{\lambda(1)}\right|+\cdots+\left|v_{\lambda(p)}\right|}\left[U\left(v_{\lambda(1)}, \ldots, v_{\lambda(p)}\right), U\left(v_{\lambda(p+1)}, \ldots, v_{\lambda(k)}\right)\right]_{G},
\end{gathered}
$$

where $\varepsilon_{\lambda}$ is defined in (4.13), $[,]_{G}$ is the Gerstenhaber bracket (2.10), and $m$ is the $A_{\infty^{-}}$ structure on $A$ obtained from $Q^{\mathfrak{o}}$, that is

$$
m\left(a_{1}, a_{2}, \ldots, a_{n}\right)=Q^{\mathfrak{o}}\left(a_{1}, a_{2}, \ldots, a_{n}\right) .
$$

Now we define the desired $L_{\infty}$-morphism from $V$ to $C^{\bullet}(A, A)$ by the formula:

$$
U\left(v_{1}, \ldots, v_{k}\right)\left(a_{1}, \ldots, a_{n}\right)=Q^{\mathfrak{o}}\left(v_{1}, \ldots, v_{k} ; a_{1}, \ldots a_{n}\right)
$$

where $v_{i} \in V$ and $a_{j} \in A$.

We claim that equation (4.17) follows from

$$
\hat{Q}^{\mathfrak{o}} \circ \hat{Q}^{\mathfrak{o}}=0 .
$$

Indeed, applying the composition $Q^{\mathfrak{o}} \circ \hat{Q}^{\mathfrak{o}}$ to the monomial

$$
\left(v_{1}, v_{2}, \ldots, v_{k}, a_{1}, a_{2}, \ldots, a_{n}\right)
$$

with $k \geq 1$ and using (4.12) we get

$$
\sum_{p=1}^{k} \sum_{\lambda \in \operatorname{Sh}_{p, k-p}}(-1)^{\varepsilon_{\lambda}} Q^{\mathfrak{o}}\left(Q^{\mathfrak{c}}\left(v_{\lambda(1)}, \ldots, v_{\lambda(p)}\right), v_{\lambda(p+1)}, \ldots, v_{\lambda(k)} ; a_{1}, \ldots, a_{n}\right)+
$$

$\sum_{1 \leq t \leq s \leq n}^{0 \leq p \leq k} \sum_{\lambda \in \operatorname{Sh}_{p, k-p}}(-1)^{\varepsilon_{\lambda}+\varepsilon_{t, p}^{\lambda}} Q^{\mathfrak{o}}\left(v_{\lambda(1)}, \ldots, v_{\lambda(p)}, ; a_{1}, \ldots, Q^{\mathfrak{o}}\left(v_{\lambda(p+1)}, \ldots, v_{\lambda(k)} ; a_{t}, \ldots, a_{s}\right), \ldots, a_{n}\right)+$ 
$\sum_{1 \leq t \leq n+1}^{0 \leq p \leq k-1} \sum_{\lambda \in \operatorname{Sh}_{p, k-p}}(-1)^{\varepsilon_{\lambda}+\varepsilon_{t, p}^{\lambda}} Q^{\mathfrak{o}}\left(v_{\lambda(1)}, \ldots, v_{\lambda(p)}, ; a_{1}, \ldots, a_{t-1}, Q^{\mathfrak{o}}\left(v_{\lambda(p+1)}, \ldots, v_{\lambda(k)}\right), a_{t}, \ldots, a_{n}\right)$, where $\varepsilon_{\lambda}$ and $\varepsilon_{t, p}^{\lambda}$ are defined in (4.13) and (4.14), respectively.

It is not hard to see that the first sum in (4.21) coincides with the left hand side of (4.17) . Furthermore, combining all the terms with $p=0$ or $p=k$ in the second sum of (4.21) and all the terms with $p=0$ in the third sum of (4.21) we get

$$
\left[m, U\left(v_{1}, \ldots, v_{k}\right)\right]_{G}\left(a_{1}, \ldots, a_{n}\right)
$$

Combining the remaining terms in the second sum and in the third sum of (4.21) we get

$$
\sum_{p=1}^{k-1} \sum_{\lambda \in \operatorname{Sh}_{p, k-p}}(-1)^{\varepsilon_{\lambda}+\left|v_{\lambda(1)}\right|+\cdots+\left|v_{\lambda(p)}\right|}\left[U\left(v_{\lambda(1)}, \ldots, v_{\lambda(p)}\right), U\left(v_{\lambda(p+1)}, \ldots, v_{\lambda(k)}\right)\right]_{G} .
$$

Thus we proved that a codifferential $\hat{Q}$ on the coalgebra $\mathbf{S}(V, A)$ gives us a $\mathbf{G e r}_{\infty}$-algebra structure on $V$, an $A_{\infty}$-structure on $A$, and an $L_{\infty}$-morphism from $V$ to the $\Lambda$ Lie-algebra $C^{\bullet}(A, A)$.

Furthermore, given a $\operatorname{Ger}_{\infty}$-algebra structure $\mathcal{D}$ on $V$, an $A_{\infty}$-structure $m$ on $A$, and an $L_{\infty}$-morphism $U$ from $V$ to $C^{\bullet}(A, A)$ the formulas

$$
Q^{\mathfrak{c}}=\mathcal{D}
$$

(4.18), (4.19) define a degree 1 codifferential $\hat{Q}$ on $\mathbf{S}(V, A)$.

Theorem 4.1 is proved.

\section{1 $\operatorname{Ger}_{\infty}^{+}$versus the operad $\mathcal{O C} \infty$ governing open-closed homotopy algebras (OCHA)}

Inspired by Zwiebach's open-closed string field theory [48] H. Kajiura and J. Stasheff introduced in [27] open-closed homotopy algebras (OCHA). These homotopy algebras were studied in papers [23], [24], [28], and [29]. In this subsection we show that the operad $\mathrm{Ger}_{\infty}^{+}$ is a natural extension of the operad $\mathcal{O C}_{\infty}$.

To recall the definition of OCHA we introduce $\mathcal{O C}^{\vee}$-coalgebras.

Definition 4.2 An $\mathcal{O C}^{\vee}$-coalgebra is a pair of a $\Lambda^{2}$ cocomm-coalgebra $V$ and a $\Lambda$ coassoccoalgebra $A$ together with a unary operation

$$
\rho: A \rightarrow V
$$

of degree -1 such that the following diagrams commute:

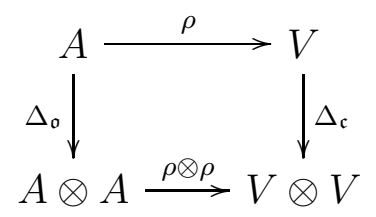




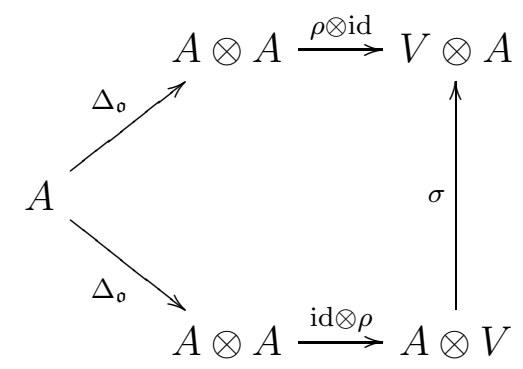

where $\Delta_{\mathfrak{c}}\left(\right.$ resp. $\left.\Delta_{\mathfrak{o}}\right)$ is the comultiplication in $V$ (resp. in A) and

$$
\sigma: A \otimes V \rightarrow V \otimes A
$$

is the mapping which switches tensor components.

We denote by $\mathcal{O} \mathcal{C}^{\vee}$ the cooperad which governs $\mathcal{O C}^{\vee}$-coalgebras.

The operad $\mathcal{O C} \mathcal{C}_{\infty}$ governing OCHA algebras is by definition the cobar construction of the cooperad $\mathcal{O} \mathcal{C}^{\vee}$.

$$
\mathcal{O C}_{\infty}=\operatorname{Cobar}\left(\mathcal{O C}^{\vee}\right)
$$

In other words, an OCHA algebra structure on a pair of graded vector spaces $(V, A)$ is a degree 1 codifferential on the free $\mathcal{O C}^{\vee}$ - coalgebra $\mathcal{O} \mathcal{C}^{\vee}(V, A)$.

Comparing Definitions 3.1 and 4.2 it is not hard to see that $\mathcal{O C}^{\vee}$ is a sub-cooperad of $\mathbf{S}$. Hence $\mathcal{O C}_{\infty}$ is a sub DG operad of $\mathrm{Ger}_{\infty}^{+}$:

$$
\mathcal{O C}_{\infty} \hookrightarrow \operatorname{Ger}_{\infty}^{+}
$$

Furthermore, Theorem 4.1 is a generalization of the following statement from [28]

Theorem 4.3 (H. Kajiura, J. Stasheff, [28]) An $\mathcal{O C}_{\infty}$-algebra structure on the pair of cochain complexes $(V, A)$ is a triple:

- $A \Lambda \mathbf{L i e}_{\infty}$-structure on $V$,

- An $A_{\infty}$-structure on $A$, and

- An $L_{\infty}$-morphism from $V$ to the Hochschild cochain complex $C^{\bullet}(A, A)$ of the $A_{\infty}$ algebra $A$.

Example 4.4 For every $A_{\infty}$-algebra $A$ the pair $\left(C^{\bullet}(A, A), A\right)$ carries a tautological OCHA structure: the $\Lambda \mathbf{L i e}_{\infty}$-structure on $C^{\bullet}(A, A)$ is simply the $\Lambda$ Lie-algebra structure given by the Hochschild differential (2.13) and the Gerstenhaber bracket (2.10); the $A_{\infty}$-structure on $A$ is the given one; finally, the $L_{\infty}$-morphism from $C^{\bullet}(A, A)$ to $C^{\bullet}(A, A)$ is the identity map.

\subsection{Homotopies of $\mathrm{Ger}_{\infty}^{+}$-algebra structures}

Let $(V, A)$ be a pair of cochain complexes. Let $\hat{Q}$ and $\hat{Q}^{\prime}$ be $\mathbf{G e r}_{\infty}^{+}$-algebra structures on $(V, A)$.

Definition 4.5 We say that the $\mathbf{G e r}_{\infty}^{+}$-algebra structures $\hat{Q}$ and $\hat{Q}^{\prime}$ are homotopic if there exists a degree 0 coderivation $\hat{\psi}$ of the coalgebra $\mathbf{S}(V, A)$ satisfying

$$
\hat{Q}^{\prime}=\exp ([\hat{\psi},]) \hat{Q}
$$


Remark 4.6 Homotopic Ger $_{\infty}^{+}$-structures on a pair $(V, A)$ are obviously quasi-isomorphic. Namely, a $\mathbf{G e r}_{\infty}^{+}$quasi-isomorphism from $(V, A, \hat{Q})$ to $\left(V, A, \hat{Q}^{\prime}\right)$ is given by the $\mathbf{S}$-coalgebra automorphism $\exp (\hat{\psi})$ of $\mathbf{S}(V, A)$.

Remark 4.7 Definition 4.5] is justified by Proposition 4.10 from paper 4 [5] by J. Chuang and $\mathrm{A}$. Lazarev. Indeed, let $\operatorname{End}_{(V, A)}$ be the endomorphism operad of the pair $(V, A)$. Then Proposition 4.10 from [5] implies that $\mathbf{G e r}_{\infty}^{+}$-algebra structures $\hat{Q}$ and $\hat{Q}^{\prime}$ are in the relation (4.26) if and only if the corresponding maps of DG operads

$$
\operatorname{Cobar}(\mathbf{S}) \rightarrow \operatorname{End}_{(V, A)}
$$

are homotopic with the homotopy defined via a path object.

We will be interested in homotopies between $\mathbf{G e r}_{\infty}^{+}$-algebra structures for which the coderivation

$$
\hat{\psi} \in \operatorname{Coder}(\mathbf{S}(V, A))
$$

satisfies the following additional conditions

$$
\begin{aligned}
& \left.\hat{\psi}\right|_{\operatorname{Ger}^{\vee}(V)}=0 . \\
& \left.\hat{\psi}\right|_{\underline{T}\left(\mathbf{s}^{-1} A\right)}=0 .
\end{aligned}
$$

These conditions are motivated by the following obvious proposition:

Proposition 4.8 Let $(V, A)$ be a pair of cochain complexes and $\hat{Q}, \hat{Q}^{\prime}$ be $\mathbf{G e r}_{\infty}^{+}$-algebra structures on $(V, A)$ satisfying equation (4.26). If the coderivation $\hat{\psi}$ enjoys condition (4.27) then the $\mathbf{G e r}_{\infty}$-algebra structures on $V$ corresponding to $\hat{Q}$ and $\hat{Q}^{\prime}$ coincide. If $\hat{\psi}$ enjoys condition (4.28) then the $A_{\infty}$-algebra structures on $A$ corresponding to $\hat{Q}$ and $\hat{Q}^{\prime}$ coincide.

Proof. The first statement follows from the fact that the $\mathbf{G e r}_{\infty}$-algebra structure on $V$ corresponding to $\hat{Q}$ is given by the c-component (4.1) of $\hat{Q}$. The second statement follows from the fact that the $A_{\infty}$-algebra structure on $A$ corresponding to $\hat{Q}$ is obtained by restricting $Q^{\mathfrak{o}}(4.5)$ to

$$
\underline{T}\left(\mathbf{s}^{-1} A\right) \subset \mathbf{s}^{-1} \mathbf{S}^{\mathfrak{o}}(V, A) .
$$

Let $\mathcal{L}$ and $\widetilde{\mathcal{L}}$ be $\Lambda$ Lie $_{\infty}$-algebras.

Recall from [8] and [37] that $L_{\infty}$-morphisms from $\mathcal{L}$ to $\widetilde{\mathcal{L}}$ can be identified with MaurerCartan elements of the auxiliary $L_{\infty}$-algebra:

$$
\mathbf{s H o m}\left(\mathbf{s}^{2} \underline{S}\left(\mathbf{s}^{-2} \mathcal{L}\right), \widetilde{\mathcal{L}}\right) .
$$

The $L_{\infty}$-structure on (4.29) is defined in terms of the $\Lambda \mathbf{L i e}_{\infty}$-structure on $\widetilde{\mathcal{L}}$, the comultiplication and the codifferential on $\mathbf{s}^{2} \underline{S}\left(\mathbf{s}^{-2} \mathcal{L}\right)$. In particular, if $\widetilde{\mathcal{L}}$ is a $\Lambda$ Lie-algebra then (4.29) is a DG Lie algebra. This is an important observation because in our consideration below $\widetilde{\mathcal{L}}$ is the Hochschild cochain complex $C^{\bullet}(A, A)$ and hence a $\Lambda$ Lie-algebra.

\footnotetext{
${ }^{4}$ I would like to thank Bruno Vallette for showing me this paper.
} 
It was also shown in [8] and [37] that homotopic $L_{\infty}$-morphisms correspond to isomorphid 5 Maurer-Cartan elements in (4.29).

We will need the following theorem.

Theorem 4.9 Let $(V, A)$ be a pair of cochain complexes and $\hat{Q}, \hat{Q}^{\prime}$ be $\mathbf{G e r}_{\infty}^{+}$-algebra structures on $(V, A)$ with coinciding $\mathbf{G e r}_{\infty}$-structures on $V$ and coinciding $A_{\infty}$-structures on $A$. Let $\hat{U}$ and $\hat{U}^{\prime}$ be $L_{\infty}$-morphisms from $V$ to $C^{\bullet}(A, A)$ corresponding to $\mathbf{G e r}_{\infty}^{+}$-algebra structures $\hat{Q}$ and $\hat{Q}^{\prime}$, respectively. Then the $L_{\infty}$-morphisms $\hat{U}$ and $\hat{U}^{\prime}$ are homotopic if and only if $\hat{Q}$ and $\hat{Q}^{\prime}$ satisfy equation (4.26) with a coderivation $\hat{\psi}$ enjoying conditions (4.27) and (4.28).

Proof. We start with the part "if".

Let $U$ (resp. $U^{\prime}$ ) be the composition of $\hat{U}$ (resp. $\hat{U}^{\prime}$ ) with the natural projection $p$ : $\mathbf{s}^{2} \underline{S}\left(\mathbf{s}^{-2} C^{\bullet}(A, A)\right) \rightarrow C^{\bullet}(A, A)$

$$
U, U^{\prime}: \mathbf{s}^{2} \underline{S}\left(\mathbf{s}^{-2} V\right) \rightarrow C^{\bullet}(A, A) .
$$

Following [8], [37] we view the maps $U$ and $U^{\prime}$ as degree 1 elements of the following auxiliary DG Lie algebra

$$
\mathcal{H}=\mathbf{s H o m}\left(\mathbf{s}^{2} \underline{S}\left(\mathbf{s}^{-2} V\right), C^{\bullet}(A, A)\right) .
$$

We need to show that there exists a degree zero element

$$
\theta \in \operatorname{sHom}\left(\mathbf{s}^{2} \underline{S}\left(\mathbf{s}^{-2} V\right), C^{\bullet}(A, A)\right)
$$

such that

$$
U^{\prime}=\exp \left([\theta,]_{\mathcal{H}}\right) U+\frac{\exp \left([\theta,]_{\mathcal{H}}\right)-1}{[\theta,]_{\mathcal{H}}} d_{\mathcal{H}} \theta
$$

where $d_{\mathcal{H}}$ and $[,]_{\mathcal{H}}$ are the differential and the bracket in $\mathcal{H}$ (4.31), respectively.

We will show that the desired element $\theta$ can be expressed in terms of the map

$$
\psi=p \circ \hat{\psi}
$$

where $p$ is the natural projection $p: \mathbf{S}(V, A) \rightarrow V \oplus A$. More precisely, for $v_{i} \in V$ and $a_{j} \in A$ we set

$$
\theta\left(v_{1}, \ldots, v_{k}\right)\left(a_{1}, \ldots, a_{n}\right)=\psi\left(v_{1}, \ldots, v_{k} ; a_{1}, \ldots, a_{n}\right) .
$$

It is easy to check that $\theta$ has the degree 0 in (4.31).

To prove (4.33) we introduce an auxiliary variable $t$ and the following path of $L_{\infty}$ morphisms

$$
U_{t}=\exp \left(t[\theta,]_{\mathcal{H}}\right) U+\frac{\exp \left(t[\theta,]_{\mathcal{H}}\right)-1}{[\theta,]_{\mathcal{H}}} d_{\mathcal{H}} \theta .
$$

Similarly, we introduce a path $\hat{Q}_{t}$ of $\mathbf{G e r}_{\infty}^{+}$-algebra structures on the pair $(V, A)$

$$
\hat{Q}_{t}=\exp (t[\hat{\psi},]) \hat{Q}
$$

Let $\widetilde{U}_{t}$ be the $L_{\infty}$-morphism from $V$ to $C^{\bullet}(A, A)$ corresponding to $\hat{Q}_{t}$. Namely,

$$
\widetilde{U}_{t}\left(v_{1}, \ldots, v_{k}\right)\left(a_{1}, \ldots, a_{n}\right)=Q_{t}\left(v_{1}, \ldots, v_{k} ; a_{1}, \ldots, a_{n}\right),
$$

\footnotetext{
${ }^{5}$ We think of Maurer-Cartan elements as objects of the corresponding Deligne groupoid [17, [18.
} 
where $Q_{t}$ is the composition $p \circ \hat{Q}_{t}$ of $\hat{Q}_{t}$ with the natural projection $p: \mathbf{S}(V, A) \rightarrow V \oplus A$.

The codifferential $\hat{Q}_{t}$ satisfies the differential equation

$$
\frac{d}{d t} \hat{Q}_{t}=\left[\hat{\psi}, \hat{Q}_{t}\right]
$$

Hence the composition $Q_{t}=p \circ \hat{Q}_{t}$ satisfies the equation

$$
\frac{d}{d t} Q_{t}=\psi \circ \hat{Q}_{t}-Q_{t} \circ \hat{\psi}
$$

Combining this equation with (4.34) and (4.37) it is not hard to show that $\widetilde{U}_{t}$ satisfies the differential equation

$$
\frac{d}{d t} \widetilde{U}_{t}=d_{\mathcal{H}} \theta+\left[\theta, \widetilde{U}_{t}\right]_{\mathcal{H}}
$$

where $d_{\mathcal{H}}$ and $[,]_{\mathcal{H}}$ are the differential and the bracket in $\mathcal{H}$ (4.31), respectively.

On the other hand, $U_{t}$ (4.35) satisfies the same differential equation (4.40) with the same initial condition

$$
\left.U_{t}\right|_{t=0}=\left.\widetilde{U}_{t}\right|_{t=0}=U
$$

Thus equation (4.33) holds and the desired implication is proved.

The proof of "only if" part is similar.

We define the map

$$
\psi: \mathbf{S}(V, A) \rightarrow V \oplus A
$$

by setting

$$
\left.\psi\right|_{\operatorname{Ger}^{\vee}(V)}=0
$$

and

$$
\psi\left(v_{1}, v_{2}, \ldots, v_{k}, a_{1}, a_{2}, \ldots a_{n}\right)=\left\{\begin{array}{l}
\theta\left(v_{1}, v_{2}, \ldots, v_{k}\right)\left(a_{1}, a_{2}, \ldots a_{n}\right), \text { if } k \geq 1 \\
0, \text { otherwise }
\end{array}\right.
$$

Next, we let $\hat{\psi}$ be the coderivation corresponding to $\psi$.

Then using a similar line of arguments it is not hard to deduce equation (4.26) from (4.33).

\subsection{Transfer theorem}

Let us now show that $\mathrm{Ger}_{\infty}^{+}$-algebra structures can be transferred across quasi-isomorphisms. More precisely,

Theorem 4.10 Given quasi-isomorphisms of cochain complexes

$$
V \rightarrow V^{\prime} \quad A \rightarrow A^{\prime}
$$

and a $\mathbf{G e r}_{\infty}^{+}$-algebra structure on the pair $\left(V^{\prime}, A^{\prime}\right)$ one can construct a $\mathbf{G e r}_{\infty}^{+}$-algebra structure on $(V, A)$ and a $\mathbf{G e r}_{\infty}^{+}$quasi-isomorphism

$$
\hat{T}:(V, A) \succ \rightarrow\left(V^{\prime}, A^{\prime}\right)
$$

which extends (4.43). 
Remark 4.11 The $\mathbf{G e r}_{\infty}^{+}$-algebra structure obtained in this way on pair $(V, A)$ is called the transferred $\mathbf{G e r}_{\infty}^{+}$-algebra structure.

Remark 4.12 We would like to remark that OCHA structures can also be transferred across quasi-isomorphisms. The corresponding transfer theorem was proved in [27, Section 4].

Proof. The construction of the transferred $\mathbf{G e r}_{\infty}^{+}$-algebra structure goes along the lines of the proof of Lemma 4.2.1 in 21 .

A $\mathrm{Ger}_{\infty}^{+}$-algebra structure on $\left(V^{\prime}, A^{\prime}\right)$ is given by a degree 1 codifferential $\hat{Q}^{\prime}$ on the S-coalgebra

$$
\mathbf{S}\left(V^{\prime}, A^{\prime}\right) \text {. }
$$

Thus we need to build a coderivation $\hat{Q}$ on the $\mathbf{S}$-coalgebra $\mathbf{S}(V, A)$ together with an $\mathbf{S}$ coalgebra homomorphism

$$
\hat{T}: \mathbf{S}(V, A) \rightarrow \mathbf{S}\left(V^{\prime}, A^{\prime}\right)
$$

such that $\hat{T}$ extends (4.43) $\hat{Q}$ extends the differential on $V \oplus A$ and

$$
\hat{Q}^{2}=0, \quad \hat{T} \hat{Q}=\hat{Q}^{\prime} \hat{T} .
$$

Following V. Hinich [21] these equations can be solved by induction on an increasing filtration on the cooperad $\mathbf{S}$. However, unlike in the proof of Lemma 4.2.1 from [21] we cannot use the filtration by arity because $\mathbf{S}$ has a non-identity unary operation $\rho$.

So we assign the following weights to the elementary cooperations of $\mathbf{S}$

$$
w(\rho)=1, \quad w\left(\Delta_{\mathfrak{o}}\right)=1, \quad w\left(\Delta_{\mathfrak{c}}\right)=2, \quad w\left(\delta_{\mathfrak{c}}\right)=2,
$$

where $\Delta_{\mathfrak{c}}\left(\operatorname{resp} . \delta_{\mathfrak{c}}\right)$ is the comultiplication (resp. cobracket) of Ger ${ }^{\vee}$ and $\Delta_{\mathfrak{o}}$ is the comultiplication of $\Lambda$ coassoc .

Since all the defining relations of the cooperad $\mathbf{S}$ are homogenous with respect to the weights (4.45) we use them to build the desired increasing filtration $F^{\bullet} \mathbf{S}$ on $\mathbf{S}$

$$
F^{1} \mathbf{S} \subset F^{2} \mathbf{S} \subset F^{3} \mathbf{S} \subset \ldots
$$

The cooperad $\mathbf{S}$ is obviously cocomplete with respect to this filtration. Furthermore, the filtration is compatible with the coinsertions $\Delta_{k}$ of the cooperad $\mathbf{S}$ in the following sense 6

$$
\Delta_{k}\left(F^{n} \mathbf{S}\right) \subset \bigoplus_{p+q=n} F^{p} \mathbf{S} \otimes F^{q} \mathbf{S}
$$

It is this property that allows us to solve equations (4.44) inductively following the lines of the proof of Lemma 4.2.1 from [21].

Remark 4.13 The minimal model condition (4.47) implies that the operad $\mathrm{Ger}_{\infty}^{+}$is cofibrant in the sense of Remark 2.1.

Remark 4.14 The transfer theorem is a common feature of homotopy algebras. As far as we know, the first example of such a theorem was proved by T. Kadeishvili in [25] for $A_{\infty}$-algebras. In this context, we would also like to mention a very interesting paper [33] by P. van der Laan. In this paper the author established koszulity for the colored operad which governs operads. Using this result he introduced the notion of operads up to homotopy and gave an elegant proof of the transfer theorem for homotopy algebras.

\footnotetext{
${ }^{6}$ This is also known as the minimal model condition [39].
} 


\section{4 $\operatorname{Ger}_{\infty}^{+}$-algebra structure on $\left(C^{\bullet}(A, A), A\right)$}

Let $A$ be an $A_{\infty}$-algebra. Following D. Tamarkin [21], [40] the Hochschild cochain complex $C^{\bullet}(A, A)$ of $A$ carries a $\mathbf{G e r}_{\infty}$-algebra structure with the following properties

$\mathbf{H}$ the induced Gerstenhaber algebra structure on the cohomology groups $H^{\bullet}\left(C^{\bullet}(A, A)\right)$ coincides with the one given by the Gerstenhaber bracket and the cup-product.

O all operations of this $\mathbf{G e r}_{\infty}$-algebra structure are expressed in terms of insertions of cochains into a cochain, insertions of cochains into $A_{\infty}$ operations, and insertions of $A_{\infty}$-operations into a cochain.

Remark 4.15 Tamarkin's Ger $_{\infty}$-algebra structure on $C^{\bullet}(A, A)$ can be obtained in two ways. The first way is to combine a solution of the Deligne conjecture [1], [32], [35], [43], [45] for Hochschild cochains with the formality for the operad of little discs [31], [34], 41]. The second way is to use the Etingof-Kazhdan theory [12] as in [44]. Both constructions involve various choices. For example, for both constructions we need to choose Drinfeld's associator $\Phi$ [11]. Following D. Tamarkin [42, different choices of the Drinfeld associator give different homotopy classes of the $\operatorname{Ger}_{\infty}$-structure on $C^{\bullet}(A, A)$.

Let us prove that

Theorem 4.16 Tamarkin's $\mathbf{G e r}_{\infty}$-algebra structure on $C^{\bullet}(A, A)$ extends to a $\mathbf{G e r}_{\infty}^{+}$-algebra structure on the pair $\left(C^{\bullet}(A, A), A\right)$.

Proof. According to Theorem 4.1 we need to construct a $\mathbf{G e r}_{\infty}$-algebra structure on $C^{\bullet}(A, A)$, an $A_{\infty}$-algebra structure on $A$ and an $L_{\infty}$-morphism from $C^{\bullet}(A, A)$ to the $\Lambda$ Liealgebra $C^{\bullet}(A, A)$.

The first two ingredients are already at hand. So we set the last one to be the identity map

$$
\text { id }: C^{\bullet}(A, A) \rightarrow C^{\bullet}(A, A) .
$$

We would like to emphasize that the $\Lambda \mathbf{L i e}_{\infty}$-algebra structure on the source of (4.48) comes from Tamarkin's $\mathbf{G e r}_{\infty}$-algebra structure while the target of (4.48) carries the $\Lambda$ Lie-algebra structure given by the Hochschild differential (2.13) and the Gerstenhaber bracket (2.10). Due to Theorem] 2 from [10] these two $\Lambda \mathbf{L i e}_{\infty}$-algebra structures on $C^{\bullet}(A, A)$ coincide. Hence, the map (4.48) is indeed an $L_{\infty}$-morphism from the $\Lambda \operatorname{Lie}_{\infty}$-algebra $C^{\bullet}(A, A)$ to the $\Lambda$ Lie-algebra of Hochschild cochains of $A$.

Remark 4.17 It is easy to see that the $\mathbf{G e r}_{\infty}^{+}$structure on the pair $\left(C^{\bullet}(A, A), A\right)$ extends the tautological OCHA structure from Example 4.4.

Let us describe the $\mathbf{G e r}_{\infty}^{+}$-algebra structure from Theorem 4.16 explicitly in the case when $A$ is a plain associative algebra concentrated in degree 0 .

\footnotetext{
${ }^{7}$ In paper [10, Theorem 2 was proved for the case of (strict) associative algebra $A$ only. However it is not hard to extend the proof from [10] to the case of an arbitrary $A_{\infty}$-algebra using the same degree bookkeeping argument.
} 
We need to define a codifferential $\hat{Q}$ on the $\mathbf{S}$-coalgebra $\mathbf{S}\left(C^{\bullet}(A, A), A\right)$. This codifferential is uniquely determined by its composition

$$
Q=p \circ \hat{Q}
$$

with the projection

$$
p: \mathbf{S}\left(C^{\bullet}(A, A), A\right) \rightarrow C^{\bullet}(A, A) \oplus A
$$

The c-component $Q^{\mathfrak{c}}$ of $Q$

$$
Q^{\mathfrak{c}}: \operatorname{Ger}^{\vee}\left(C^{\bullet}(A, A)\right) \rightarrow C^{\bullet}(A, A)
$$

is Tamarkin's $\mathbf{G e r}_{\infty}$-algebra structure on $C^{\bullet}(A, A)$ and the $\mathfrak{o}$-component is defined as follows.

For $a_{1}, \ldots, a_{n} \in A$ and $P, P_{1}, \ldots, P_{k} \in C^{\bullet}(A, A)$ we set

$$
\begin{gathered}
Q^{\mathfrak{o}}\left(a_{1}, a_{2}, \ldots, a_{n}\right)=\left\{\begin{array}{l}
-a_{1} a_{2}, \text { if } n=2, \\
0, \text { otherwise },
\end{array}\right. \\
Q^{\mathfrak{o}}\left(P_{1}, P_{2} \ldots, P_{k} ; a_{1}, a_{2}, \ldots, a_{n}\right)=0
\end{gathered}
$$

if $k>1$ and

$$
Q^{\mathfrak{o}}\left(P ; a_{1}, a_{2}, \ldots, a_{n}\right)=\left\{\begin{array}{l}
P\left(a_{1}, \ldots, a_{n}\right), \text { if }|P|=n \\
0, \text { otherwise } .
\end{array}\right.
$$

In particular, for $b \in C^{0}(A)$ we have

$$
Q^{\mathfrak{o}}(b)=b,
$$

where, in the right hand side, $b$ is viewed as an element of $A$.

\section{The formality quasi-isomorphism for $C^{\bullet}(A, A)$ via trans- fer of the $\mathrm{Ger}_{\infty}^{+}$-structure}

Let $A$ be a commutative unital algebra over the field $\mathbb{K}$ and let

$$
V_{A}^{\bullet}=\wedge_{A}^{\bullet} \operatorname{Der}(A)
$$

be the graded algebra of polyvector fields on $\operatorname{Spec}(A)$. It is known that $V^{\bullet}(A)$ is a Gerstenhaber algebra. The multiplication is simply the exterior product and the Lie bracket is the Schouten-Nijenhuis bracket $[,]_{S N}$.

Let

$$
\hat{Q}_{H}: \mathbf{S}\left(V_{A}^{\bullet}, A\right) \rightarrow \mathbf{S}\left(V_{A}^{\bullet}, A\right)
$$

be a $\mathbf{G e r}_{\infty}^{+}$-structure on the pair $\left(V_{A}^{\bullet}, A\right)$. As above we denote by $Q_{H}$ the composition $p \circ \hat{Q}_{H}$ of $\hat{Q}_{H}$ with the natural projection:

$$
p: \mathbf{S}\left(V_{A}^{\bullet}, A\right) \rightarrow V_{A}^{\bullet} \oplus A .
$$

We also denote by $Q_{H}^{\mathfrak{c}}$ and $Q_{H}^{\mathfrak{o}}$ the $\mathfrak{c}$-component and $\mathfrak{o}$-component, respectively

$$
\begin{gathered}
Q_{H}^{\mathfrak{c}}: \operatorname{Ger}^{\vee}\left(V_{A}^{\bullet}\right) \rightarrow V_{A}^{\bullet}, \\
Q_{H}^{\mathfrak{o}}: \mathbf{s}\left(\underline{S}\left(\mathbf{s}^{-2} V_{A}^{\bullet}\right) \otimes T\left(\mathbf{s}^{-1} A\right)\right) \oplus \mathbf{s} \underline{T}\left(\mathbf{s}^{-1} A\right) \rightarrow A .
\end{gathered}
$$

Theorem 4.1 implies that 
Corollary 5.1 If a $\mathbf{G e r}_{\infty}^{+}$algebra structure (5.2) on the pair $\left(V_{A}^{\bullet}, A\right)$ satisfies these two properties:

$\mathbf{G}$ the $\mathbf{G e r}_{\infty}$-structure on $V_{A}^{\bullet}$ is the standard Gerstenhaber algebra structure given by the Schouten-Nijenhuis bracket and the exterior product;

A the $A_{\infty}$-algebra structure on $A$ is the original associative algebra structure on $A$ then the formula

$$
F\left(\gamma_{1}, \ldots, \gamma_{k}\right)\left(a_{1}, \ldots, a_{n}\right)=Q_{H}^{\mathfrak{o}}\left(\gamma_{1}, \ldots, \gamma_{k} ; a_{1}, \ldots, a_{n}\right)
$$

defines an $L_{\infty}$-morphism from the $\Lambda$ Lie-algebra $V_{A}^{\bullet}$ to the $\Lambda$ Lie-algebra $C^{\bullet}(A, A)$.

Conversely, if

$$
\hat{F}: V_{A}^{\bullet} \succ \rightarrow C^{\bullet}(A, A)
$$

is an $L_{\infty}$-morphism from the $\Lambda$ Lie-algebra $V_{A}^{\bullet}$ to $C^{\bullet}(A, A)$ then the formula (5.3) (with $F=p \circ \hat{F})$ defines a $\mathbf{G e r}_{\infty}^{+}$-structure on the pair $\left(V_{A}^{\bullet}, A\right)$ satisfying the above properties $\mathbf{G}$ and $\mathbf{A}$.

We will denote the $\mathbf{G e r}_{\infty}^{+}$-structure on the pair $\left(V_{A}^{\bullet}, A\right)$ corresponding to an $L_{\infty}$-morphism (5.4) by $\hat{Q}_{\hat{F}}$.

Remark 5.2 An OCHA analogue of Corollary 5.1 was already proved in [28]. In fact, J. Kajiura and J. Stasheff considered in [28] the OCHA structure on the pair $\left(V_{A}^{\bullet}, A\right)$ for $A=\mathbb{R}\left[x^{1}, \ldots, x^{d}\right]$ corresponding to Kontsevich's formality quasi-isomorphism from [30] .

Let us now restrict our consideration to the case when $A$ is a regular commutative algebra. (In other words, for every prime ideal $\mathfrak{p} \subset A$ the local ring $A_{\mathfrak{p}}$ is regular.) In this case, the Hochschild-Kostant-Rosenberg theorem [22] tells us that the Hochschild cohomology $H H^{\bullet}(A, A)$ is isomorphic to the Gerstenhaber algebra (5.1) of polyvector fields on $\operatorname{Spec}(A)$.

For regular commutative algebras we also have the following theorem.

Theorem 5.3 (Theorem 4, [10]) Let D be Tamarkin's $\mathbf{G e r}_{\infty}$-structure on $C^{\bullet}(A, A)$. Then for every regular commutative algebra $A$ (over $\mathbb{K}$ ) there exists a $\mathbf{G e r}_{\infty}$ quasi-isomorphism

$$
\hat{G}: V_{A}^{\bullet} \succ \rightarrow C^{\bullet}(A, A)
$$

from the Gerstenhaber algebra $V_{A}^{\bullet}$ of polyvector fields to the $\operatorname{Ger}_{\infty}$-algebra $\left(C^{\bullet}(A, A), \mathcal{D}\right)$.

Due to Theorem 2 from [10] the $\Lambda$ Lie $\infty_{\infty}$-part of the $\mathbf{G e r}_{\infty}$-structure $\mathcal{D}$ on $C^{\bullet}(A, A)$ coincides with the standard $\Lambda$ Lie-algebra structure on $C^{\bullet}(A, A)$. Therefore, restricting the $\mathbf{G e r}_{\infty^{-}}$ quasi-isomorphism $\hat{G}$ to the cocommutative coalgebra

$$
\Lambda^{2} \operatorname{cocomm}\left(V_{A}^{\bullet}\right)=\mathbf{s}^{2} \underline{S}\left(\mathbf{s}^{-2} V_{A}^{\bullet}\right)
$$

we get an $L_{\infty}$ quasi-isomorphism

$$
\left.\hat{G}\right|_{\mathbf{s}^{2} S\left(\mathbf{s}^{-2} V_{\dot{A}}^{\bullet}\right.} \mathbf{s}^{2} S\left(\mathbf{s}^{-2} V_{A}^{\bullet}\right) \rightarrow \mathbf{s}^{2} S\left(\mathbf{s}^{-2} C^{\bullet}(A, A)\right)
$$

from the $\Lambda$ Lie-algebra $V_{A}^{\bullet}$ to the $\Lambda$ Lie-algebra $C^{\bullet}(A, A)$.

The following theorem addresses the question of whether a given $L_{\infty}$-morphism $\hat{F}$ (5.4) is homotopic to the $L_{\infty}$ quasi-isomorphism (5.6). 
Theorem 5.4 Let $\hat{Q}_{\hat{F}}$ be the $\mathbf{G e r}_{\infty}^{+}$-structure on the pair $\left(V_{A}^{\bullet}, A\right)$ corresponding to an $L_{\infty}$ morphism $\hat{F}$ (5.4). Furthermore, let $\hat{Q}$ be the $\operatorname{Ger}_{\infty}^{+}$-structure on the pair $\left(C^{\bullet}(A, A), A\right)$ which extends Tamarkin's $\mathbf{G e r}_{\infty}$-structure $\mathcal{D}$ on $C^{\bullet}(A, A)$ via Theorem 4.16. Then the $L_{\infty}$ morphism $\hat{F}$ is homotopic to the $L_{\infty}$ quasi-isomorphism (5.6) if and only if the $\mathbf{G e r}_{\infty}^{+}$ algebras $\left(V_{\hat{A}}^{\bullet}, A, \hat{Q}_{\hat{F}}\right)$ and $\left(C^{\bullet}(A, A), A, \hat{Q}\right)$ are quasi-isomorphic.

Proof. We start with the part "only if".

Let $\hat{F}$ be homotopic to the $L_{\infty}$-quasi-isomorphism (5.6). Due to Theorem 4.9 and Remark 4.6 we may assume, without loss of generality, that $\hat{F}$ coincides with an $L_{\infty}$-quasiisomorphism (5.6). In other words,

$$
\left.\hat{G}\right|_{\mathbf{s}^{2} \underline{S}\left(\mathbf{s}^{-2} V_{\dot{A}}^{\bullet}\right)}=\hat{F} .
$$

Then we define a morphism $\hat{T}$ from the $\mathbf{S}$-coalgebra $\mathbf{S}\left(V_{\dot{A}}^{\bullet}, A\right)$ to $\mathbf{S}\left(C^{\bullet}(A, A), A\right)$ by setting

$$
\left.\hat{T}\right|_{\operatorname{Ger}^{\vee}\left(V_{\dot{A}}^{\bullet}\right)}=\hat{G}
$$

and

$$
T^{\mathfrak{o}}\left(\gamma_{1}, \ldots, \gamma_{k}, a_{1}, \ldots, a_{n}\right)=\left\{\begin{array}{l}
a_{1} \text { if } k=0 \text { and } n=1 \\
0, \text { otherwise }
\end{array}\right.
$$

where

$$
T^{\mathfrak{o}}: \mathbf{s}\left(\underline{S}\left(\mathbf{s}^{-2} V_{A}^{\bullet}\right) \otimes T\left(\mathbf{s}^{-1} A\right)\right) \oplus \mathbf{s} \underline{T}\left(\mathbf{s}^{-1} A\right) \rightarrow A
$$

is the $\mathfrak{o}$-component of the composition $T=p \circ \hat{T}$.

Equations (5.7) and (5.8) imply that

$$
F\left(\gamma_{1}, \ldots, \gamma_{k}\right)=T^{\mathfrak{c}}\left(\gamma_{1}, \ldots, \gamma_{k}\right)
$$

for all $\gamma_{i} \in V_{\dot{A}}^{\bullet}$. Here $T^{\mathfrak{c}}$ denotes the $\mathfrak{c}$-component of the composition $T=p \circ \hat{T}$ and $F$ is the composition $F=p \circ \hat{F}$.

We claim that $\hat{T}$ is a $\mathbf{G e r}_{\infty}^{+}$quasi-isomorphism from $\mathbf{G e r}_{\infty}^{+}$-algebra $\left(V_{\hat{A}}^{\bullet}, A, \hat{Q}_{\hat{F}}\right)$ to the $\operatorname{Ger}_{\infty}^{+}$-algebra $\left(C^{\bullet}(A, A), A, \hat{Q}\right)$. In other words,

$$
\hat{Q} \circ \hat{T}=\hat{T} \circ \hat{Q}_{\hat{F}} .
$$

Indeed, the $\mathfrak{c}$-component of this equation

$$
\left.\hat{Q} \circ \hat{T}\right|_{\operatorname{Ger}^{\vee}(V)}=\left.\hat{T} \circ \hat{Q}_{\hat{F}}\right|_{\operatorname{Ger}^{\vee}(V)}
$$

holds because $\hat{G}$ is a $\mathbf{G e r}_{\infty}$-quasi-isomorphism.

To show that the $\mathfrak{o}$-component of (5.11) holds it suffices to prove that

$$
Q^{\mathfrak{o}} \circ \hat{T}^{\mathfrak{o}}\left(\gamma_{1}, \ldots, \gamma_{k} ; a_{1}, a_{2}, \ldots, a_{n}\right)=T^{\mathfrak{o}} \circ \hat{Q}_{\hat{F}}^{\mathfrak{o}}\left(\gamma_{1}, \ldots, \gamma_{k} ; a_{1}, a_{2}, \ldots, a_{n}\right) .
$$

The latter equation is a consequence of (4.50), (4.51), (4.52), (5.9), and (5.10).

The part "if" requires more work. 
Let $\hat{T}: \mathbf{S}\left(V_{A}^{\bullet}, A\right) \rightarrow \mathbf{S}\left(C^{\bullet}(A, A), A\right)$ be a morphism of $\mathbf{S}$-coalgebras compatible with the codifferentials $\hat{Q}_{\hat{F}}$ on $\mathbf{S}\left(V_{A}^{\bullet}, A\right)$ and $\hat{Q}$ on $\mathbf{S}\left(C^{\bullet}(A, A), A\right)$. As above $T$ is the composition $p \circ \hat{T}$ with the natural projection $p: \mathbf{S}\left(C^{\bullet}(A, A), A\right) \rightarrow C^{\bullet}(A, A) \oplus A$ and $T^{\mathfrak{c}}$ and $T^{\mathfrak{o}}$ are the $\mathfrak{c}$-component and the $\mathfrak{o}$-component of $T$, respectively.

Since the c-component $\hat{T}^{\mathfrak{c}}$

$$
\hat{T}^{\mathfrak{c}}: \operatorname{Ger}^{\vee}\left(V_{A}^{\bullet}\right) \rightarrow \operatorname{Ger}^{\vee}\left(C^{\bullet}(A, A)\right)
$$

is a $\operatorname{Ger}_{\infty}$ quasi-isomorphism from $V_{A}^{\bullet}$ to the $\operatorname{Ger}_{\infty}$-algebra $\left(C^{\bullet}(A, A), \mathcal{D}\right)$, we simply need to show that $\hat{F}$ (5.4) is homotopic to the $L_{\infty}$ quasi-isomorphism

$$
\left.\hat{T}^{\mathrm{c}}\right|_{\mathbf{s}^{2} \underline{S}\left(\mathbf{s}^{-2} V_{\dot{A}}^{\bullet}\right)}: \mathbf{s}^{2} \underline{S}\left(\mathbf{s}^{-2} V_{A}^{\bullet}\right) \rightarrow \mathbf{s}^{2} \underline{S}\left(\mathbf{s}^{-2} C^{\bullet}(A, A)\right) .
$$

Since $A$ has the zero differential and it is concentrated in the single degree 0 we have

$$
T^{\mathfrak{o}}\left(a_{1}\right)=a_{1}
$$

and

$$
T^{\mathfrak{o}}\left(a_{1}, \ldots, a_{n}\right)=0
$$

for all $n>1$ and $a_{i} \in A$.

Unfolding the o-component of the equation $\hat{Q} \circ \hat{T}=\hat{T} \circ \hat{Q}_{\hat{F}}$ we get

$$
\begin{gathered}
Q^{\mathfrak{o}} \circ \hat{T}^{\mathfrak{o}}\left(\gamma_{1}, \ldots, \gamma_{k} ; a_{1}, a_{2}, \ldots, a_{n}\right)= \\
\sum_{p=1}^{k} \sum_{\lambda \in \operatorname{Sh}_{p, k-p}}(-1)^{\varepsilon_{\lambda}} T^{\mathfrak{o}}\left(Q_{\hat{F}}^{\mathfrak{c}}\left(\gamma_{\lambda(1)}, \ldots, \gamma_{\lambda(p)}\right), \gamma_{\lambda(p+1)}, \ldots, \gamma_{\lambda(k)} ; a_{1}, \ldots, a_{n}\right)+ \\
\sum_{1 \leq t \leq s \leq n}^{0 \leq p \leq k} \sum_{\lambda \in \operatorname{Sh}_{p, k-p}}(-1)^{\varepsilon_{\lambda}+\varepsilon_{t, p}^{\lambda}} T^{\mathfrak{o}}\left(\gamma_{\lambda(1)}, \ldots, \gamma_{\lambda(p)} ; a_{1}, \ldots, Q_{\hat{F}}^{\mathfrak{o}}\left(\gamma_{\lambda(p+1)}, \ldots, \gamma_{\lambda(k)} ; a_{t}, \ldots, a_{s}\right) \ldots, a_{n}\right)+ \\
\sum_{1 \leq t \leq n+1}^{0 \leq p-1} \sum_{\lambda \in \operatorname{Sh}_{p, k-p}}(-1)^{\varepsilon_{\lambda}+\varepsilon_{t, p}^{\lambda}} T^{\mathfrak{o}}\left(\gamma_{\lambda(1)}, \ldots, \gamma_{\lambda(p)}, ; a_{1}, \ldots, a_{t-1}, Q_{\hat{F}}^{\mathfrak{o}}\left(\gamma_{\lambda(p+1)}, \ldots, \gamma_{\lambda(k)}\right), a_{t}, \ldots, a_{n}\right)
\end{gathered}
$$

where

$$
\begin{gathered}
\varepsilon_{\lambda}=\sum_{i<j}\left|\gamma_{i}\right|\left|\gamma_{j}\right|, \\
\varepsilon_{t, p}^{\lambda}=\sum_{j=1}^{p}\left|\gamma_{\lambda(j)}\right|+\sum_{j=p+1}^{k} \sum_{l=1}^{t-1}\left|\gamma_{\lambda(j)}\right|\left(\left|a_{l}\right|+1\right)+\sum_{l=1}^{t-1}\left(\left|a_{l}\right|+1\right) .
\end{gathered}
$$

In the case $k=1$ equation (5.16) reduces to

$$
\begin{gathered}
-T^{\mathfrak{o}}\left(\gamma, a_{1}, \ldots, a_{n-1}\right) a_{n}-(-1)^{|\gamma|} a_{1} T^{\mathfrak{o}}\left(\gamma, a_{2}, \ldots, a_{n}\right)+T^{\mathfrak{c}}(\gamma)\left(a_{1}, \ldots, a_{n}\right)= \\
F(\gamma)\left(a_{1}, \ldots, a_{n}\right)-(-1)^{|\gamma|} T^{\mathfrak{o}}\left(\gamma, a_{1} a_{2}, a_{3}, \ldots, a_{n}\right)+(-1)^{|\gamma|} T^{\mathfrak{o}}\left(\gamma, a_{1}, a_{2} a_{3}, \ldots, a_{n}\right)+\ldots \\
+(-1)^{|\gamma|+n-1} T^{\mathfrak{o}}\left(\gamma, a_{1}, \ldots, a_{n-2}, a_{n-1} a_{n}\right) .
\end{gathered}
$$


Here we use (4.50), (4.51), (4.52), (5.14), and (5.15).

A simple degree bookkeeping shows that if $|\gamma| \neq n$ then all terms of equation (5.19) are identically zero.

Thus equation (5.19) is equivalent to

$$
T^{\mathfrak{c}}(\gamma)=F(\gamma)+\left[m, \psi_{1}(\gamma)\right]_{G}
$$

where $m$ is the multiplication in $A$ and $\psi_{1}$ is a map from $V_{A}^{\bullet}$ to $C^{\bullet}(A, A)$ of degree -1 defined by the formula

$$
\psi_{1}(\gamma)\left(a_{1}, a_{2}, a_{3}, \ldots, a_{|\gamma|-1}\right)=T^{\mathfrak{o}}\left(\gamma, a_{1}, a_{2}, a_{3}, \ldots, a_{|\gamma|-1}\right) .
$$

Equation (5.20) implies that $F$ is an $L_{\infty}$ quasi-isomorphism. Furthermore, substituting $F$ by a homotopic $L_{\infty}$ quasi-isomorphism we can "kill" $\psi_{1}$.

In other words, with loss of generality, we may assume that $\psi_{1}=0$ and hence

$$
T^{\mathfrak{o}}\left(\gamma, a_{1}, a_{2}, a_{3}, \ldots, a_{s}\right)=0
$$

and

$$
T^{\mathfrak{c}}(\gamma)=F(\gamma)
$$

for all $\gamma \in V_{A}^{\bullet}$ and $a_{i} \in A$.

Let us assume, by induction, that for all $k^{\prime}<k$

$$
T^{\mathfrak{o}}\left(\gamma_{1}, \ldots, \gamma_{k^{\prime}} ; a_{1}, \ldots, a_{n}\right)=0
$$

and

$$
T^{\mathfrak{c}}\left(\gamma_{1}, \ldots, \gamma_{k^{\prime}}\right)=F\left(\gamma_{1}, \ldots, \gamma_{k^{\prime}}\right)
$$

for all $\gamma_{i} \in V_{A}^{\bullet}$ and $a_{j} \in A$.

Then equation (5.16) reduces to

$$
\begin{gathered}
-T^{\mathfrak{o}}\left(\gamma_{1}, \ldots, \gamma_{k} ; a_{1}, \ldots, a_{n-1}\right) a_{n}-(-1)^{\left|\gamma_{1}\right|+\cdots+\left|\gamma_{k}\right|} a_{1} T^{\mathfrak{o}}\left(\gamma_{1}, \ldots, \gamma_{k} ; a_{2}, \ldots, a_{n}\right)+ \\
T^{\mathfrak{c}}\left(\gamma_{1}, \ldots, \gamma_{k}\right)\left(a_{1}, \ldots, a_{n}\right)=F\left(\gamma_{1}, \ldots, \gamma_{k}\right)\left(a_{1}, \ldots, a_{n}\right)- \\
(-1)^{\left|\gamma_{1}\right|+\cdots+\left|\gamma_{k}\right|}\left(T^{\mathfrak{o}}\left(\gamma_{1}, \ldots, \gamma_{k} ; a_{1} a_{2}, a_{3}, \ldots, a_{n}\right)-T^{\mathfrak{o}}\left(\gamma_{1}, \ldots, \gamma_{k} ; a_{1}, a_{2} a_{3}, \ldots, a_{n}\right)+\ldots\right. \\
\left.+T^{\mathfrak{o}}\left(\gamma_{1}, \ldots, \gamma_{k} ; a_{1}, \ldots, a_{n-2}, a_{n-1} a_{n}\right)\right) .
\end{gathered}
$$

Here we use (4.50), (4.51), (4.52) and (5.23).

Again, a simple degree bookkeeping shows that if $n \neq\left|\gamma_{1}\right|+\cdots+\left|\gamma_{k}\right|+2-2 k$ then all terms of equation (5.25) are identically zero.

Thus equation (5.25) is equivalent to

$$
T^{\mathfrak{c}}\left(\gamma_{1}, \ldots, \gamma_{k}\right)=F\left(\gamma_{1}, \ldots, \gamma_{k}\right)+\left[m, \psi_{k}\left(\gamma_{1}, \ldots, \gamma_{k}\right)\right]_{G}
$$

where $m$ is the multiplication in $A$ and $\psi_{k}$ is a map from $S^{k}\left(V_{A}^{\bullet}\right)$ to $C^{\bullet}(A, A)$ of degree $1-2 k$ defined by the formula

$$
\psi_{k}\left(\gamma_{1}, \ldots, \gamma_{k}\right)\left(a_{1}, \ldots, a_{n-1}\right)=T^{\mathfrak{o}}\left(\gamma_{1}, \ldots, \gamma_{k} ; a_{1}, \ldots, a_{n-1}\right)
$$

and $n=\left|\gamma_{1}\right|+\cdots+\left|\gamma_{k}\right|+2-2 k$. 
Combining this observation with the inductive assumption (5.24) we see that substituting $\hat{F}$ by a homotopic $L_{\infty}$ quasi-isomorphism we can "kill" $\psi_{k}$ and hence adjust $\hat{T}$ in such a way that

$$
T^{\mathfrak{o}}\left(\gamma_{1}, \ldots, \gamma_{k}, a_{1}, \ldots, a_{n}\right)=0
$$

and

$$
T^{\mathfrak{c}}\left(\gamma_{1}, \ldots, \gamma_{k}\right)=F\left(\gamma_{1}, \ldots, \gamma_{k}\right)
$$

for all $\gamma_{i} \in V_{A}^{\bullet}$ and $a_{j} \in A$.

This argument completes the step of the induction and hence the proof of the theorem.

Let us now restrict our consideration to the case when $A$ is the polynomial algebra

$$
A=\mathbb{K}\left[x^{1}, \ldots, x^{d}\right]
$$

in $d$ variables. In this case the Gerstenhaber algebra structure on $V_{A}^{\bullet}$ satisfies the following rigidity property:

Theorem 5.5 (Tamarkin's rigidity, [21], [40]) Let $\mathbb{K}$ be a field of characteristic zero. If $A=\mathbb{K}\left[x^{1}, x^{2}, \ldots, x^{d}\right]$ then any $\mathbf{G e r}_{\infty}$-algebra structure on $V_{A}^{\bullet}$ with the binary operations $\wedge$ and $[,]_{S N}$ is homotopy equivalent to the Gerstenhaber algebra $\left(V_{A}^{\bullet}, \wedge,[,]_{S N}\right)$.

For the proof of this theorem we refer the reader to Section 5.4 of nice exposition [21] by V. Hinich.

Applying Theorem 4.10 to the $\mathbf{G e r}_{\infty}^{+}$-algebra structure on the pair $\left(C^{\bullet}(A, A), A\right)$ we get a transferred $\mathbf{G e r}_{\infty}^{+}$-algebra structure on the cohomology $\left(V_{A}^{\bullet}, A\right)$.

This $\mathbf{G e r}_{\infty}^{+}$-algebra structure automatically satisfies Property A from Corollary [5.1. Indeed, this fact follows easily from the observation that $A$ is concentrated in the single degree 0 .

Property G from Corollary 5.1 is not satisfied in general. However, using Theorem 5.5 we can adjust the transferred $\mathbf{G e r}_{\infty}^{+}$-algebra structure $\hat{Q}_{H}$ on $\left(V_{A}^{\bullet}, A\right)$ so that it will satisfy Property G. It is clear that Property A will still be satisfied for this new $\mathbf{G e r}_{\infty}^{+}$-algebra structure on $\left(V_{A}^{\bullet}, A\right)$.

Thus we obtained a $\mathbf{G e r}_{\infty}^{+}$-algebra structure $\hat{Q}_{H}$ on the pair $\left(V_{A}^{\bullet}, A\right)$ which satisfies Properties $\mathbf{G}$ and $\mathbf{A}$ from Corollary 5.1. In other words, $\hat{Q}_{H}$ defines (and is defined by) an $L_{\infty}$-morphism

$$
\hat{F}: V_{A}^{\bullet} \succ \rightarrow C^{\bullet}(A, A) .
$$

In addition, the $\mathbf{G e r}_{\infty}^{+}$-algebra $\left(V_{A}^{\bullet}, A, \hat{Q}_{H}\right)$ is quasi-isomorphic to $\left(C^{\bullet}(A, A), A, \hat{Q}\right)$. Thus Theorem 5.4 implies the following corollary

Corollary 5.6 Let $A=\mathbb{K}\left[x^{1}, \ldots, x^{d}\right]$. If

$$
\hat{F}: V_{A}^{\bullet} \succ \rightarrow C^{\bullet}(A, A)
$$

is an $L_{\infty}$-morphism obtained, as above, via transfer of the $\mathbf{G e r}_{\infty}^{+}$-structure on $\left(C^{\bullet}(A, A), A\right)$ to the cohomology then $\hat{F}$ is homotopic to an $L_{\infty}$ quasi-isomorphism constructed via Theorem 5.3 . 


\section{A Link between $\mathrm{Ger}_{\infty}^{+}$and Voronov's Swiss Cheese operad SC}

In this section we describe a link between the DG operad $\mathrm{Ger}_{\infty}^{+}$and the Fulton-MacPherson version SC of Voronov's Swiss Cheese operad [46].

Let us first describe the 2-colored topological operad SC .

\subsection{The Swiss Cheese operad SC}

To define the spaces of the 2-colored operad SC we introduce the configuration space Conf $k$ of $k$ labeled distinct points on the complex plane $\mathbb{C}$. Similarly, we introduce the configuration space $\operatorname{Conf}_{k, n}$ of $k+n$ labeled distinct points on $\mathbb{C}$ such that the first $k$ points lie on the upper half plane

$$
\mathbf{H}=\{z \in \mathbb{C} \mid \operatorname{Im}(z)>0\}
$$

and the last $n$ points lie on the real line $\mathbb{R}$. Next, we define the space $C_{k}$ as the quotient of Conf $_{k}$ by the action of the 3-dimensional real Lie group

$$
z \mapsto a z+b, \quad a \in \mathbb{R}, a>0, \quad b \in \mathbb{C} .
$$

Similarly, $C_{k, n}$ is the quotient of $\operatorname{Conf}_{k, n}$ by the action of the 2-dimensional real Lie group:

$$
z \mapsto a z+b, \quad a \in \mathbb{R}, a>0, \quad b \in \mathbb{R}
$$

The spaces $\operatorname{SC}^{\mathfrak{c}}(k, n)$ are empty whenever $n \geq 1$,

$$
\operatorname{SC}^{\mathfrak{c}}(k, 0):=\bar{C}_{k},
$$

and

$$
\mathrm{SC}^{\mathfrak{o}}(k, n):=\bar{C}_{k, n},
$$

where $\bar{C}_{k}$ and $\bar{C}_{k, n}$ are respectively compactifications of $C_{k}$ and $C_{k, n}$ constructed by $\mathrm{M}$. Kontsevich in [30, Section 5].

The spaces $\bar{C}_{k}$ and $\bar{C}_{k, n}$ are obtained from $C_{k}$ and $C_{k, n}$, respectively, à la FultonMacPherson by performing real blow-ups along all partial diagonals 8

These spaces are manifolds with corners and open strata of $\bar{C}_{k}$ are in correspondence with $k$-labeled planar trees. More precisely, given a $k$-labeled planar tree $T$ with the set of vertices $V(T)$, a stratum $C_{T}$ corresponding to $T$ is isomorphic to the following product

$$
C_{T}=\prod_{v \in V(T) \backslash\{\text { leaves }\}} C_{k(v)},
$$

where $k(v)$ is the number of incoming 9 edges of the vertex $v$. The planar tree $T$ tells us in which order we need to perform blow-ups to get the stratum $C_{T}$.

\footnotetext{
${ }^{8}$ Building $\bar{C}_{k, n}$ we should also perform real blow-ups along the pieces of the boundary which correspond to the situation when a cluster of points in the upper half plane approaches the real line $\mathbb{R}$.

${ }^{9}$ We tacitly assume that all trees are rooted and hence oriented: each vertex has exactly one outgoing edge and may have several incoming edges; a vertex with no incoming edges is called a leaf.
} 
The correspondence between planar trees and strata of $\bar{C}_{k}$ is not bijective. For example, if two $k$-labeled planar trees $T$ and $T^{\prime}$ give the same $k$-labeled tree then we identify the strata $C_{T}$ and $C_{T^{\prime}}$ by relabeling points in components $C_{k(v)}, v \in V(T) \backslash$ leaves $\}$ according to the difference in the planar structures of $T$ and $T^{\prime}$.

To describe strata of $\bar{C}_{k, n}$ we need to use 2-colored planar trees. A 2-colored planar tree $T$ is a planar tree $T$ together with a map

$$
\chi: E(T) \rightarrow\{\mathfrak{c}, \mathfrak{o}\}
$$

which assigns to each edge $e \in E(T)$ a color $\mathfrak{c}$ or a color $\mathfrak{o}$. We require that the total order $\{\mathfrak{c}<\mathfrak{o}\}$ on the set $\{\mathfrak{c}, \mathfrak{o}\}$ is compatible with the planar structure in the following sense: for every vertex $v$ of the tree $T$ the restriction of the map $\chi$ to the set $E_{i n}(v)$ of edges terminating at the vertex $v$ is order preserving. In other words, if we walk around the vertex $v$ in the clockwise direction starting with the outgoing edge then we first cross all the edges in $E_{\text {in }}(v)$ with the color $\mathfrak{c}$ and then all the edges in $E_{i n}(v)$ with the color $\mathfrak{o}$. For an illustration, see the left picture on figure 1 .
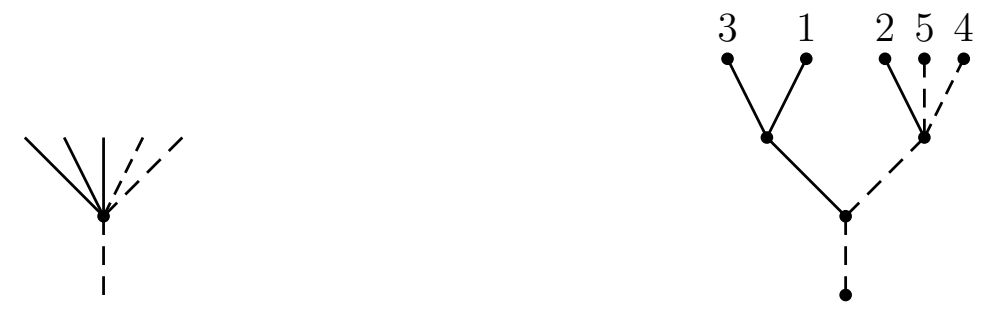

Fig. 1: Dashed edges have the color $\mathfrak{o}$, solid edges have the color $\mathfrak{c}$

For our purposes we also impose the following condition on a 2-colored planar tree $T$ :

Condition 6.1 If at least one incoming edge of a vertex $v$ has the color $\mathfrak{o}$ then its outgoing edge has the color $\mathfrak{o}$.

For every 2-colored planar tree $T$ the set $V(T)$ of vertices splits into the disjoint union according to the color of the outgoing edge

$$
V(T)=V_{\mathfrak{c}}(T) \sqcup V_{\mathfrak{o}}(T) .
$$

In other words, a vertex $v$ belongs to $V_{\mathfrak{c}}(T)$ (resp. $V_{\mathfrak{o}}(T)$ ) if its outgoing edge has the color $\mathfrak{c}$ (resp. the color $\mathfrak{o})$. The above condition implies that for every vertex $v \in V_{\mathfrak{c}}(T)$ all its incoming edges have the color $\mathfrak{c}$.

A $(k, n)$-labeled 2-colored planar tree $T$ is a 2-colored planar tree $T$ equipped with a bijection between the set of leaves and the set $\{1,2, \ldots, k+n\}$ such that the external edges corresponding to the first $k$ leaves have the color $\mathfrak{c}$ and the external edges corresponding to the last $n$ leaves have the color $\mathfrak{o}$. For an example of a $(3,2)$-labeled 2-colored planar tree see the right picture on figure 1 .

We can now describe open strata of $\bar{C}_{k, n}$. A stratum $C_{T}$ of $\bar{C}_{k, n}$ corresponding to a $(k, n)$-labeled 2-colored planar tree $T$ is the following product

$$
C_{T}=\prod_{v \in V_{\mathfrak{c}}(T) \backslash\{\text { leaves }\}} C_{k(v)} \times \prod_{v \in V_{\mathfrak{o}}(T) \backslash\{\text { leaves }\}} C_{k(v), n(v)},
$$


where $k(v)$ (resp. $n(v)$ ) denotes the number of incoming edges with color $\mathfrak{c}$ (resp. with color $\mathfrak{o})$.

Remark 6.2 Condition 6.1 implies that the root edge of every $(k, n)$-labeled 2-colored planar tree $T$ with $n \neq 0$ has necessarily the color $\mathfrak{o}$. In the case $n=0$ we require, in addition, that the root of $T$ in (6.5) has the color $\mathfrak{o}$. For example, we may have a $(3,0)$-labeled 2-colored planar tree depicted on figure 2 .

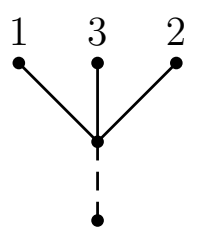

Fig. 2: The dashed edge has the color $\mathfrak{o}$, solid edges have the color $\mathfrak{c}$

As well as for $\bar{C}_{k}$ the correspondence between planar 2-colored trees and strata of $\bar{C}_{k, n}$ is not bijective. For example, if two $(k, n)$-labeled 2-colored planar trees $T$ and $T^{\prime}$ give the same $(k, n)$-labeled 2-colored tree then we identify the strata $C_{T}$ and $C_{T^{\prime}}$ by relabeling points in components $C_{k(v)}, v \in V_{\mathfrak{c}}(T) \backslash$ \{leaves $\}$ and $C_{k(v), n(v)}, v \in V_{\mathfrak{o}}(T) \backslash$ \{leaves $\}$ according to the difference in the planar structures of $T$ and $T^{\prime}$.

The operadic insertions for SC are defined in the obvious way using concatenation of trees and identification of the corresponding strata.

Following this description, it is not hard to see that, in the category of sets, SC is the free 2-colored operad generated by the collection $\left\{C_{k}, C_{k, n}\right\}$ where $C_{k}$ 's give us operations of arity

$$
(\underbrace{\mathfrak{c}, \ldots, \mathfrak{c}}_{k} \mapsto \mathfrak{c})
$$

and $C_{k, n}$ 's give us operations of arity

$$
(\underbrace{\mathfrak{c}, \ldots, \mathfrak{c}}_{k}, \underbrace{\mathfrak{o}, \ldots, \mathfrak{o}}_{n} \mapsto \mathfrak{o})
$$

\subsection{The operad $H_{\bullet}(\mathrm{SC})$ and the cooperads $H^{\bullet}(\mathrm{SC})$ and sc}

The homology operad $H_{\bullet}(\mathrm{SC})$ was described in [46]:

Theorem 6.3 (A. Voronov, [46]) Algebras over $H_{\bullet}(\mathrm{SC})$ are triples $(V, A, \varrho)$, where $V$ is a Gerstenhaber algebra, $A$ is an associative algebra and

$$
\varrho: V \rightarrow A
$$

is a homomorphism of associative algebras satisfying the condition

$$
\varrho(v) a=(-1)^{|v||a|} a \varrho(v)
$$

for all $v \in V$ and $a \in A$. 
Proof. Although a proof is given in [46] we would like to briefly explain how one can prove this statement without using the original (non-Fulton-MacPherson) version of the Swiss Cheese operad.

Spaces of the operad SC are the compatifications $\bar{C}_{k}$ and $\bar{C}_{k, n}$ described above. The top dimensional stratum of $\bar{C}_{k}$ (resp. $\bar{C}_{k, n}$ ) is the configuration space $C_{k}$ (resp. $C_{k, n}$ ). Thus, by the collar neighborhood theorem, one can construct a homotopy equivalence between $\bar{C}_{k}$ (resp. $\bar{C}_{k, n}$ ) and $C_{k}$ (resp. $\left.C_{k, n}\right)$. Hence

$$
H_{\bullet}\left(\bar{C}_{k}\right) \cong H_{\bullet}\left(C_{k}\right), \quad H_{\bullet}\left(\bar{C}_{k, n}\right) \cong H_{\bullet}\left(C_{k, n}\right) .
$$

The homology of the spaces $C_{k}$ and $C_{k, n}$ can be computed along the lines of [6] or [19]. Finally, using the homotopy equivalence between $\bar{C}_{k}\left(\operatorname{resp} . \bar{C}_{k, n}\right)$ and $C_{k}\left(\operatorname{resp} . C_{k, n}\right)$ it is not hard to see how the desired operad assembles from the homology spaces $H_{\bullet}\left(C_{k}\right)$ and $H_{\bullet}\left(C_{k, n}\right)$.

Remark 6.4 Using the collar neighborhood theorem again (or applying the universal coefficient theorem) we get the similar description for the spaces of the linear dual cooperad $H^{\bullet}(\mathrm{SC})$ :

$$
H^{\bullet}\left(\bar{C}_{k}\right) \cong H^{\bullet}\left(C_{k}\right) \quad H^{\bullet}\left(\bar{C}_{k, n}\right) \cong H^{\bullet}\left(C_{k, n}\right) .
$$

The cooperad $H^{\bullet}$ (SC) is closely related to the cooperad $\mathbf{S}$ (see Definition 3.1). To describe a link between these two cooperads we introduce the following modification of $H^{\bullet}(\mathrm{SC})$ coalgebras:

Definition 6.5 An sc-coalgebra is a pair of $\mathbf{G e r}^{\vee}$-coalgebra $V$ and a $\Lambda$ coassoc-coalgebra $A$ together with a unary operation

$$
\rho: A \rightarrow V
$$

of degre $10-1$ such that the following diagrams commute:
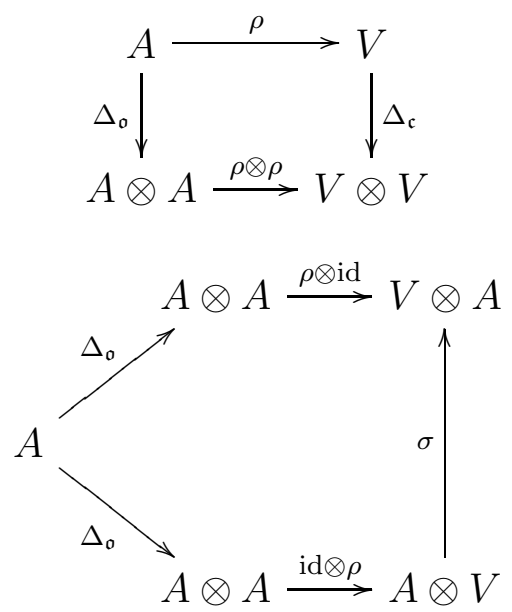

where $\Delta_{\mathfrak{c}}\left(\right.$ resp. $\left.\Delta_{\mathfrak{o}}\right)$ is the comultiplication in $V$ (resp. in A) and

$$
\sigma: A \otimes V \rightarrow V \otimes A
$$

is the mapping which switches tensor components. We will denote by $\mathbf{s c}$ the 2-colored cooperad which governs sc-coalgebras.

\footnotetext{
${ }^{10}$ Recall that by degree of $\rho$ we mean the degree of the corresponding vector in the cooperad governing sc-coalgebras.
} 
Remark 6.6 It is clear that the cooperad sc is obtained from $H^{\bullet}(\mathrm{SC})$ by shifting the degrees of the cooperations $\Delta_{\mathfrak{c}}, \delta_{\mathfrak{c}}, \rho, \Delta_{\mathfrak{o}}$ by $-2,-2,-1$, and -1 , respectively. In other words, we have

$$
\mathbf{s c}^{\mathfrak{c}}(k, 0)=\mathbf{s}^{2-2 k} H^{\bullet}(\mathrm{SC})^{\mathfrak{c}}(k, 0), \quad \mathbf{s c}^{\mathfrak{o}}(k, n)=\mathbf{s}^{1-n-2 k}\left(1 \otimes \operatorname{sgn}_{n}\right) \otimes H^{\bullet}(\mathrm{SC})^{\mathfrak{o}}(k, n),
$$

where $1 \otimes \operatorname{sgn}_{n}$ is the product of the trivial representation of $S_{k}$ and the sign representation of $S_{n}$.

It is not hard to see that an $\mathbf{S}$-coalgebra $(V, A)$ is an sc-coalgebra with an additional condition:

$$
\delta_{\mathfrak{c}} \circ \rho=0,
$$

where $\delta_{\mathfrak{c}}$ denotes the Lie cobracket on $V$. Thus the cooperad $\mathbf{S}$ is a sub-cooperad of $\mathbf{s c}$

$$
\mathrm{S} \hookrightarrow \mathbf{S C}
$$

\subsection{The first sheet $E^{1}(\mathrm{SC})$ of the spectral sequence for $\mathrm{SC}$}

Using the stratification of the spaces (6.2), (6.3) described in Subsection 6.1 we equip the topological operad SC with the increasing filtration

$$
\cdots \subset F^{p} \mathrm{SC} \subset F^{p+1} \mathrm{SC} \subset \ldots
$$

where $F^{p} \mathrm{SC}$ is the closure of the union of all strata of dimension $p$.

In [46] A. Voronov considered the first sheet $E^{1}(\mathrm{SC})$ of the spectral sequence corresponding to this filtration. He referred to the DG operad $E^{1}(\mathrm{SC})$ as the operad governing "homotopy Swiss Cheese algebras". To build a link between the DG operads $\mathrm{Ger}_{\infty}^{+}$and $E^{1}(\mathrm{SC})$ we would like to show that

Theorem 6.7 The DG operads $E^{1}(\mathrm{SC})$ and Cobar(sc) are isomorphic.

Proof. The spaces $\bar{C}_{k}$ form a Fulton-MacPherson version $\mathrm{FME}_{2}$ of the little disc operad. So the operad SC is a 2-colored extension of the ordinary (non-colored) operad $\mathrm{FME}_{2}$.

Thus if we restrict our consideration to operations of $E^{1}(\mathrm{SC})$ of the arity

$$
(\mathfrak{c}, \mathfrak{c}, \ldots, \mathfrak{c} \mapsto \mathfrak{c})
$$

then we get an ordinary (non-colored) DG operad

$$
E^{1}\left(\mathrm{FME}_{2}\right)
$$

In the famous Getzler-Jones papen [19] the DG operad (6.14) was identified with the cobar construction $\operatorname{Cobar}\left(\mathbf{G e r}^{\vee}\right)$ of the cooperad $\mathbf{G e r}^{\vee}$.

Thus, to prove the theorem, it remains to extend this identification to the operations of the arity

$$
(\underbrace{\mathfrak{c}, \ldots, \mathfrak{c}}_{k}, \underbrace{\mathfrak{o}, \ldots, \mathfrak{o}}_{n} \mapsto \mathfrak{o})
$$

\footnotetext{
${ }^{11}$ See also Proposition 1.2 in [45].
} 
For $E_{\bullet, \bullet}^{1}$ of $\bar{C}_{k, n}$ we have 12

$$
E_{p, q}^{1}\left(\bar{C}_{k, n}\right)=H_{p+q}\left(F^{-p} \bar{C}_{k, n}, F^{-p-1} \bar{C}_{k, n}\right) .
$$

$F^{-p-1} \bar{C}_{k, n}$ is the boundary of the compact manifold $F^{-p} \bar{C}_{k, n}$. Hence by the PoincaréLefschetz duality we have

$$
H_{p+q}\left(F^{-p} \bar{C}_{k, n}, F^{-p-1} \bar{C}_{k, n}\right) \cong H^{q}\left(F^{-p} \bar{C}_{k, n}\right) .
$$

Next, the collar neighborhood theorem implies that the space $F^{-p} \bar{C}_{k, n}$ is homotopy equivalent to its interior

$$
F^{-p} \bar{C}_{k, n} \backslash F^{-p-1} \bar{C}_{k, n}
$$

Therefore, we have the isomorphism

$$
H^{q}\left(F^{-p} \bar{C}_{k, n}\right) \cong H^{q}\left(F^{-p} \bar{C}_{k, n} \backslash F^{-p-1} \bar{C}_{k, n}\right) .
$$

The space (6.17) is the disjoint union of strata of dimension $-p$. Hence, using (6.5) and the Künneth formula we get

$$
\begin{gathered}
H_{p+q}\left(F^{-p} \bar{C}_{k, n}, F^{-p-1} \bar{C}_{k, n}\right) \cong \\
\bigoplus_{T}\left(\bigotimes_{v \in V_{\mathrm{c}}(T) \backslash\{\text { leaves }\}} H^{\bullet}\left(C_{k(v)}\right) \otimes \bigotimes_{v \in V_{\mathrm{o}}(T) \backslash\{\text { leaves }\}} H^{\bullet}\left(C_{k(v), n(v)}\right)\right)^{q} / \sim
\end{gathered}
$$

where the summation runs over all $(k, n)$-labeled 2-colored planar trees $T$ and $k(v)$ (resp. $n(v)$ ) denotes the number of incoming edges of the vertex $v$ with the color $\mathfrak{c}$ (resp. with the color $\mathfrak{o}$ ). In the right hand side we identify two vectors if one of them is obtained from another by changing the total order on the set of the incoming edges of a vertex $v \in V_{\mathfrak{c}}(T) \backslash\{$ leaves $\}$ (resp. $v \in V_{\mathfrak{o}}(T) \backslash\{$ leaves $\}$ ) and applying the corresponding element of the symmetric group to the component in $H^{\bullet}\left(C_{k(v)}\right)$ (resp. $H^{\bullet}\left(C_{k(v), n(v)}\right)$ ).

Since the dimension of the stratum $C_{T}$ corresponding to the tree $T$ is

$$
-p=\operatorname{dim}\left(C_{T}\right)=\sum_{v \in V_{\mathfrak{c}}(T)}\left(2 k_{v}-3\right)+\sum_{v \in V_{\mathfrak{o}}(T)}\left(2 k_{v}+n_{v}-2\right)
$$

we get that the total degree of a vector in (6.19) reads:

$$
p+q=q+\sum_{v \in V_{\mathfrak{c}}(T)}\left(2-2 k_{v}\right)+\sum_{v \in V_{\mathfrak{o}}(T)}\left(1-2 k_{v}-n_{v}\right)+\left|V_{\mathfrak{c}}(T)\right|+\left|V_{\mathfrak{o}}(T)\right| .
$$

Combining this computation with (6.8) and Remark 6.6 we get the desired identification between $E^{1}(\mathrm{SC})$ and Cobar(sc) as operads of graded vector spaces.

To show that the differential $d_{1}$ in $E^{1}(\mathrm{SC})$ coincides with the differential $\partial^{\text {Cobar }}$ of the cobar construction we need to prove commutativity of the following diagram

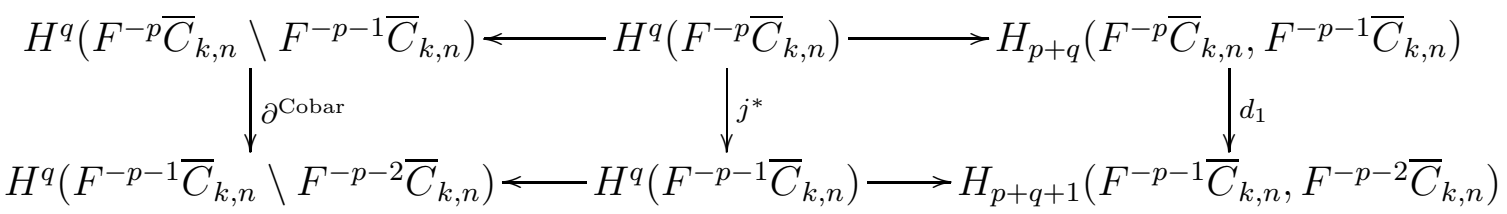

\footnotetext{
${ }^{12}$ Recall that we use the reversed grading on all homological complexes.
} 
Here $j$ is the embedding $j: F^{-p-1} \bar{C}_{k, n} \hookrightarrow F^{-p} \bar{C}_{k, n}$, the horizontal arrows in the right hand side are the cap-products with $\left[F^{-p} \bar{C}_{k, n}\right]$ and $\left[F^{-p-1} \bar{C}_{k, n}\right]$, respectively. Finally, the horizontal arrows in the left hand side are isomorphisms built using the collar neighborhood theorem.

Let us recall that the differential $d_{1}$ in $E^{1}(\mathrm{SC})$ is the composition of the coboundary operator

$$
H_{p+q}\left(F^{-p} \bar{C}_{k, n}, F^{-p-1} \bar{C}_{k, n}\right) \rightarrow H_{p+q+1}\left(F^{-p-1} \bar{C}_{k, n}\right)
$$

with the natural map

$$
H_{p+q+1}\left(F^{-p-1} \bar{C}_{k, n}\right) \rightarrow H_{p+q+1}\left(F^{-p-1} \bar{C}_{k, n}, F^{-p-2} \bar{C}_{k, n}\right)
$$

Thus the commutativity of the right half of the diagram follows from the naturality of the cap-product.

To show that the left half of the diagram (6.21) commutes we let $C_{T}$ be a top dimensional stratum of $F^{-p} \bar{C}_{k, n}$ corresponding to a 2-colored tree $T$. Then each top dimensional stratum $C_{\hat{T}}$ of the boundary $\partial C_{T}$ corresponds to a 2-colored tree $\hat{T}$ such that $T$ is obtained from $\hat{T}$ by contracting exactly one edge of $\hat{T}$. Using this observation it is not hard to see that the map $j^{*}$ is expressed in terms of cooperadic coinsertions for $H^{\bullet}(\mathrm{SC})$.

Theorem 6.7 is proved.

The embedding of cooperads (6.12) gives us the embedding of the DG operads:

$$
\operatorname{Ger}_{\infty}^{+}=\operatorname{Cobar}(\mathbf{S}) \hookrightarrow \operatorname{Cobar}(\mathbf{s c}) \cong E^{1}(\mathrm{SC}) .
$$

Thus $\mathrm{Ger}_{\infty}^{+}$is a sub DG operad of $E^{1}(\mathrm{SC})$.

Combining (6.24) with (4.25) we get the following pair of embeddings of DG operads:

$$
\mathcal{O C}_{\infty} \hookrightarrow \mathrm{Ger}_{\infty}^{+} \hookrightarrow E^{1}(\mathrm{SC})
$$

The embedding $\mathcal{O C}_{\infty} \hookrightarrow E^{1}(\mathrm{SC})$ was described in details in paper [23] by E. Hoefel.

\section{The DG operads $\mathrm{Ger}_{\infty}^{+}$and $E^{1}(\mathrm{SC})$ are non-formal}

Let us recall that the DG operad $\mathbf{G e r}_{\infty}^{+}$is the cobar construction Cobar(S) of the cooperad $\mathbf{S}$. The minimal model condition (4.47) implies that $\mathbf{G e r}_{\infty}^{+}$is cofibrant. Hence, if the operad $\mathrm{Ger}_{\infty}^{+}$is formal then there exists a quasi-isomorphism of DG operads

$$
\varphi: \operatorname{Cobar}(\mathbf{S}) \rightarrow H
$$

where $H=H^{\bullet}(\operatorname{Cobar}(\mathbf{S}))$.

Let us consider the element

$$
X=\mathbf{s}(\rho \otimes \mathrm{id}) \Delta_{\mathfrak{o}}=\mathbf{s}(\mathrm{id} \otimes \rho) \Delta_{\mathfrak{o}}
$$

in $\operatorname{Cobar}(\mathbf{S})^{\mathfrak{0}}(1,1)$.

Since $\rho$ and $\Delta_{\mathfrak{o}}$ have the degree -1 , the element $X$ has the degree -1 . It is not hard to see that $X$ spans the subspace of all operations having the arity $((\mathfrak{c}, \mathfrak{o}) \rightarrow \mathfrak{o})$ and the degree -1 . On the other hand, $X$ is not a cocycle:

$$
\partial^{\operatorname{Cobar}}(X)=\mathbf{s} \Delta_{\mathfrak{o}} \quad \circ_{1} \quad \mathbf{s} \rho+\mathbf{s} \Delta_{\mathfrak{o}} \quad \circ_{2} \quad \mathbf{s} \rho \neq 0,
$$


where $o_{1}\left(\right.$ resp. $\left.o_{2}\right)$ denotes the operadic insertion in the first slot (resp. the second slot).

Therefore, in $H$ there is no non-zero vector having the arity $((\mathfrak{c}, \mathfrak{o}) \rightarrow \mathfrak{o})$ and degree -1 . Hence, we have

$$
\varphi(X)=0 .
$$

Let $Z$ be the following element of $\operatorname{Cobar}(\mathbf{S})$

$$
Z=\mathbf{s}(\rho \otimes \rho) \Delta_{\mathfrak{o}}
$$

Due to axioms of the $\mathbf{S}$-algebra the element $Z$ can be also written as

$$
Z=\mathrm{s} \Delta_{\mathfrak{c}} \rho
$$

Next we compute $\partial^{\text {Cobar }}(Z)$

$$
\partial^{\operatorname{Cobar}}(Z)=X \quad \circ_{2} \quad \mathbf{s} \rho-X \quad \circ_{1} \quad \mathbf{s} \rho-Y,
$$

where

$$
Y=\mathbf{s} \rho{ }_{1} \quad \mathbf{s} \Delta_{\mathfrak{c}} .
$$

The element $Y$ is clearly a non-trivial cocycle in $\operatorname{Cobar}(\mathbf{S})$. On the other hand, equations (7.3) and (7.5) imply that

$$
\varphi(Y)=0
$$

Using this contradiction we deduce that the DG operad $\mathrm{Ger}_{\infty}^{+}$is non-formal. In other words

Theorem 7.1 It is not possible to construct a sequence of quasi-isomorphisms of DG operads which connects $\mathbf{G e r}_{\infty}^{+}$to its cohomology.

Using the identical line of arguments it is easy to prove that

Theorem 7.2 It is not possible to construct a sequence of quasi-isomorphisms of DG operads which connects $E^{1}(\mathrm{SC})$ to its cohomology.

\section{Discussion}

It is known [7], [36] that path integrals can be used to transfer homotopy algebraic structures. Following this line, the computations of A.S. Cattaneo and G. Felder in 4] can be considered as an example of the transfer of the tautological OCHA on $\left(C^{\bullet}(A, A), A\right)$ from Example 4.4 to its cohomology $\left(H H^{\bullet}(A, A), A\right)$ for $A=\mathbb{R}\left[x^{1}, \ldots, x^{d}\right]$.

It would be interesting to check whether the Cattaneo-Felder construction 4 can be extended further to provide us with a transfer of the $\mathbf{G e r}_{\infty}^{+}$-algebra structure on $\left(C^{\bullet}(A, A), A\right)$ to the cohomology. If this is the case then Kontsevich's formality quasi-isomorphism [30] is homotopic 13 to the one obtained via Tamarkin's procedure [10], [21], [40].

It would be also interesting to answer these questions:

\footnotetext{
${ }^{13}$ As far as the author knows, T. Willwacher [4] proved that Kontsevich's formality quasi-isomorphism is indeed homotopic to the one obtained via Tamarkin's procedure.
} 
- What is the role of the full operad $E^{1}(\mathrm{SC})$ in the construction of formality quasiisomorphisms?

- Is there a relation between the $\mathbf{G e r}_{\infty}^{+}$-algebra structure on $\left(C^{\bullet}(A, A), A\right)$ introduced in Theorem 4.16 and the solution of the Swiss Cheese conjecture proposed in [9]?

As for the first question, it is natural to guess that $E^{1}(\mathrm{SC})$-algebra structure on a pair $(V, A)$ is the following data:

1. A $\operatorname{Ger}_{\infty}$-structure on $V$,

2. an $A_{\infty}$-structure on $A$, and

3. a $\mathbf{G e r}_{\infty}$-morphism from $V$ to $C^{\bullet}(A, A)$,

where $C^{\bullet}(A, A)$ carries Tamarkin's $\mathbf{G e r}_{\infty}$-structure.

This naive guess turns out to be wrong. However, by correcting the differential $d_{1}$ on $E^{1}(\mathrm{SC})$ one can probably get algebraic structures described by the above data. Unfortunately, the author does not know any topological meaning of such corrections.

We suspect that the answer to the first question may shed some light on the second one.

\section{References}

[1] C. Berger and B. Fresse, Combinatorial operad actions on cochains, Math. Proc. Cambridge Philos. Soc., 137, 1 (2004) 135-174.

[2] C. Berger and I. Moerdijk, Axiomatic homotopy theory for operads, Comment. Math. Helv. 78, 4 (2003) 805-831; arXiv:math/0206094.

[3] C. Berger and I. Moerdijk, Resolution of coloured operads and rectification of homotopy algebras, Contemp. Math. 431 (2007) 31-58; arXiv:math/0512576.

[4] A.S. Cattaneo and G. Felder, A path integral approach to the Kontsevich quantization formula, Commun. Math. Phys. 212 (2000) 591-611; arXiv:math/9902090.

[5] J. Chuang and A. Lazarev, Feynman diagrams and minimal models for operadic algebras, arXiv:0802.3507.

[6] F. R. Cohen, The homology of $\mathcal{C}_{n+1}$-spaces, $n \geq 0$, The homology of iterated loop spaces, Springer-Verlag, 1976, Lecture Notes Math., 533, 207-351.

[7] K.J. Costello, Renormalisation and the Batalin-Vilkovisky formalism, arXiv:0706.1533.

[8] V. Dolgushev, Erratum to: "A Proof of Tsygan's Formality Conjecture for an Arbitrary Smooth Manifold", arXiv:math/0703113.

[9] V. Dolgushev, D. Tamarkin, and B. Tsygan, Proof of Swiss Cheese Version of Delignes Conjecture, accepted to IMRN; arXiv:0904.2753. 
[10] V.A. Dolgushev, D.E. Tamarkin, and B.L. Tsygan, The homotopy Gerstenhaber algebra of Hochschild cochains of a regular algebra is formal, J. Noncomm. Geom. 1, 1 (2007) 1-25; math.KT/0605141.

[11] V.G. Drinfeld, On quasitriangular quasi-Hopf algebras and on a group that is closely connected with $\operatorname{Gal}(\overline{\mathbb{Q}} / \mathbb{Q})$. (Russian) Algebra i Analiz 2, 4 (1990) 149-181; translation in Leningrad Math. J. 2, 4 (1991) 829-860.

[12] P. Etingof and D. Kazhdan, Quantization of Lie bialgebras. II, III, Selecta Math. (N.S.) 4, 2 (1998) 213-231, 233-269.

[13] B. Fresse, Koszul duality of operads and homology of partition posets, in "Homotopy theory and its applications (Evanston, 2002)", Contemp. Math. 346 (2004) 115-215.

[14] M. Gerstenhaber, The cohomology structure of an associative ring, Ann. Math. 78 (1963) 267-288.

[15] M. Gerstenhaber and A.A. Voronov, Homotopy G-algebras and moduli space operad, Internat. Math. Res. Notices, 3 (1995) 141-153.

[16] E. Getzler, Cartan homotopy formulas and the Gauss-Manin connection in cyclic homology, in Quantum deformations of algebras and their representations, Israel Math. Conf. Proc. 7 (1993) 65-78.

[17] E. Getzler, A Darboux theorem for Hamiltonian operators in the formal calculus of variations, Duke Math. J. 111, 3 (2002) 535-560.

[18] E. Getzler, Lie theory for nilpotent L-infinity algebras, Ann. of Math. (2) 170, 1 (2009) 271-301; arXiv:math/0404003.

[19] E. Getzler and J.D.S. Jones, Operads, homotopy algebra and iterated integrals for double loop spaces, hep-th/9403055.

[20] V. Ginzburg and M. Kapranov, Koszul duality for operads, Duke Math. J. 76, 1 (1994) 203-272.

[21] V. Hinich, Tamarkin's proof of Kontsevich formality theorem, Forum Math. 15, 4 (2003) 591-614; math.QA/0003052.

[22] G. Hochschild, B. Kostant, and A. Rosenberg, Differential forms on regular affine algebras, Trans. Amer. Math. Soc. 102 (1962) 383-408.

[23] E. Hoefel, OCHA and the swiss-cheese operad, J. Homotopy Relat. Struct. 4, 1 (2009) $123-151$.

[24] E. Hoefel, On the coalgebra description of OCHA, arXiv:math/0607435.

[25] T.V. Kadeishvili, The algebraic structure in the homology of an $A_{\infty}$-algebra. (Russian) Soobshch. Akad. Nauk Gruzin. SSR 108, 2 (1982) 249-252. 
[26] T.V. Kadeishvili, The structure of the $A(\infty)$-algebra, and the Hochschild and Harrison cohomologies. (Russian) Trudy Tbiliss. Mat. Inst. Razmadze. Akad. Nauk Gruzin. SSR 91 (1988) 19-27.

[27] H. Kajiura and J. Stasheff, Homotopy algebras inspired by classical open-closed string field theory, Commun. Math. Phys. 263 (2006) 553-581; arXiv:math/0410291.

[28] H. Kajiura and J. Stasheff, Open-closed homotopy algebra in mathematical physics, J. Math. Phys. 47, 2 (2006) 023506, 28 pp; arXiv:hep-th/0510118.

[29] H. Kajiura and J. Stasheff, Homotopy algebra of open-closed strings, Groups, homotopy and configuration spaces, 229-259, Geom. Topol. Monogr., 13, Geom. Topol. Publ., Coventry, 2008; arXiv:hep-th/0606283.

[30] M. Kontsevich, Deformation quantization of Poisson manifolds, Lett. Math. Phys., 66 (2003) 157-216; q-alg/9709040.

[31] M. Kontsevich, Operads and motives in deformation quantization, Lett. Math. Phys. 48 (1999) 35-72; math.QA/9904055.

[32] M. Kontsevich and Y. Soibelman, Deformations of algebras over operads and Deligne's conjecture, Conférence Moshé Flato 1999, Vol. I (Dijon), 255-307, Math. Phys. Stud., 21, Kluwer Acad. Publ., Dordrecht, 2000; math.QA/0001151.

[33] P. van der Laan, Coloured Koszul duality and strongly homotopy operads, arXiv:math/0312147.

[34] P. Lambrechts and I. Volic, Formality of the little N-disks operad, arXiv:0808.0457.

[35] J. E. McClure and J. H. Smith, A solution of Deligne's Hochschild cohomology conjecture, Contemp. Math. 293 (2002) 153-193, Amer. Math. Soc., Providence, RI; math.QA/9910126.

[36] P. Mnev, Notes on simplicial BF theory, Mosc. Math. J. 9, 2 (2009) 371-410; arXiv:hep-th/0610326.

[37] B. Shoikhet, An explicit construction of the Quillen homotopical category of dg Lie algebras, arXiv:0706.1333.

[38] J. Stasheff, Homotopy associativity of H-spaces. I, II, Trans. Amer. Math. Soc. 108 (1963) 275-292; ibid. 108 (1963) 293-312.

[39] D. Sullivan, Infinitesimal computations in topology, IHES. Publ. Math. No. 47 (1977) 269-331.

[40] D. Tamarkin, Another proof of M. Kontsevich formality theorem, math.QA/9803025.

[41] D. Tamarkin, Formality of chain operad of small squares, Lett. Math. Phys. 66, 1-2 (2003) 65-72; math.QA/9809164.

[42] D. Tamarkin, Action of the Grothendieck-Teichmüller group on the operad of Gerstenhaber algebras, arXiv:math/0202039. 
[43] D. Tamarkin, What do DG categories form? Compos. Math. 143, 5 (2007) 1335-1358; math.CT/0606553.

[44] D. Tamarkin and B. Tsygan, Non-commutative differential calculus, homotopy BV algebras and formality conjectures, Methods Funct. Anal. Topology 6, 2 (2000) 85-100; math.KT/0002116.

[45] A.A. Voronov, Homotopy Gerstenhaber algebras, Proceedings of the Moshé Flato conference, Kluwer Academic Publishers, the Netherlands, 2 (2000) 307-331; math.QA/9908040.

[46] A.A. Voronov, The Swiss-Cheese Operad, Proc. of the conference: "Homotopy invariant algebraic structures" (Baltimore, MD, 1998), 365-373, Contemp. Math., 239, Amer. Math. Soc., Providence, RI, 1999.

[47] T. Willwacher, private communication.

[48] B. Zwiebach, Oriented open-closed string theory revisited, Annals Phys. 267 (1998) 193; hep-th/9705241.

Department of Mathematics, Temple University, 1805 N. Broad St.,

Philadelphia PA, 19122 USA

E-mail address: vald@temple.edu 